\title{
Relative Hedging of Systematic Mortality Risk *
}

\author{
Erhan Bayraktar ${ }^{\dagger} \quad$ Michael Ludkovski ${ }^{\ddagger}$
}

\begin{abstract}
We study indifference pricing mechanisms for mortality contingent claims under stochastic mortality age structures. Our focus is on capturing the internal cross-hedge between components of an insurer's portfolio, especially between life annuities and life insurance. We carry out an exhaustive analysis of the dynamic exponential premium principle which is the representative nonlinear pricing rule in our framework. Along the way we also derive and compare a variety of linear pricing rules which value claims under various martingale measures. We illustrate our examples with realistic numerical examples that show the relative importance of model parameters.
\end{abstract}

Keywords: stochastic mortality, asymptotic expansions, exponential premium principle, indifference pricing

JEL Code: G11, G13, G22

\section{Introduction}

The past several decades have witnessed remarkable improvements in longevity. Over the second half of the 20th century the global life expectancy at birth increased on average by 4.5 months per year. In the USA, the average life expectancy of an individual aged 65 has increased by nearly five years during 1950-2004, while the mortality rate at age 40 has decreased by over $40 \%$. Looking ahead, the trend is expected to continue: various projections have estimated that mortality at age 80 could improve by as much as $50 \%$ over the next twenty years, and that mortality at age 70 might fall by $75 \%$ over the next 40 years (Swiss Re 2007).

Such predictions however obscure the fact that the declines in mortality have been variable over the years and across different ages. For instance an extensive analysis in Cairns et al. (2004) documents a high degree of unpredictability about future mortality, including cases of temporary mortality increases. These facts are of the greatest importance to the insurance industry that makes use of the above projections in its pricing of actuarial products. As stated in a recent report from Swiss Re (2007), "unanticipated improvements in life expectancy have a major impact on the funding required to meet pension liabilities. The biggest hurdle to estimating funding needs accurately lies in the fact that there is much uncertainty as to future longevity trends". To underscore this risk, some forecasters, such as the Mortality Investigation Bureau run by the UK Society of Actuaries, now publish multiple longevity projections.

\footnotetext{
${ }^{*}$ This research is supported by the Society of Actuaries through a CKER Research Grant

${ }^{\dagger}$ Department of Mathematics, University of Michigan, Ann Arbor, MI 48109, USA, email: erhan@umich.edu

${ }^{\ddagger}$ Department of Mathematics, University of Michigan, Ann Arbor, MI 48109, USA, email: mludkov@umich.edu
} 
The resulting adjustments in computing actuarial mortality/longevity loadings have been challenging to implement. If the mortality improvement in the population as a whole is greater than assumed, the insurer might be left with insufficient assets to cover its liabilities. This biometric risk cannot be mitigated by demographic diversification; to account for it, it is necessary to explicitly model the respective uncertainty which has been termed systematic mortality risk. On a mathematical level it is represented by stochastic fluctuations in population mortality rates over time. This has been the programme of the nascent literature on stochastic mortality (Milevsky and Promislow 2001, Dahl 2004, Cairns et al. 2004, Schrager 2006, Dahl and Møller 2006, Bayraktar and Young 2007a, Ludkovski and Young 2007, Young 2008) which uses techniques from other areas of financial mathematics to quantify this phenomenon and improve the pricing of longevity risk.

To obtain practically relevant results, a stochastic mortality model must reflect three major features of the current insurance universe: (a) non-tradable nature of mortality risk, (b) presence of many types of insurance products with different dependence on mortality rates, (c) agestructure of mortality tables. In more technical jargon, the above features mean that: (a) the stochastic mortality problem is an incomplete market, (b) one must study relative effect of mortality risk on insurer's portfolio, and (c) one must incorporate age-structure of stochastic mortality. Of course, the final aspect of the model is its practical relevance. Consequently, once a particular model is chosen and the necessary parameters are estimated, a numerical investigation is required to see the effect of the above three features on insurer's behavior. A crucial issue is the relative impact and economic significance of these ingredients, so as to find a good trade-off between model realism and tractability. While one can always build a very rich stochastic model, a practicing actuary first and foremost cares about parsimony and ease of use.

In this paper we build a mathematical framework to systematically provide qualitative analysis of these issues. Our model is quite complex but has a rigorous stochastic control approach and incorporates a fully dynamic setting. It is especially useful in providing explicit asymptotic expansions showing the impact of model parameters, including existing portfolio, stochastic mortality risk, and risk-preferences on the insurer's optimal investment. Thus we are able to disassemble the effect of each feature, as well as provide numerical illustrations of the resulting premium adjustments. Along the way we compare several different pricing mechanisms that emerge from our model and give a detailed discussion of age-distribution effects on pricing.

The rest of the paper is organized as follows. Section 2 describes the framework used for building the model and the relative exponential indifference rule used for calculating premia. Section 3 then proceeds to give the main analytic results regarding the resulting risk-adjusted prices. These are contrasted with other pricing mechanisms in Section 4 and numerically illustrated in Section 5. Section 6 is devoted to asymptotic expansions in various model parameters and explicit numerical illustrations of their impact. We then proceed in Section 7 to study the effect of mortality age-structure mentioned above. Finally, Section 8 summarizes our results and concludes.

\section{Temporary Annuities and Term Life Insurance}

For concreteness and clarity of presentation, we shall focus on a particular (and in some sense canonical) example of an insurer whose portfolio consists of term life insurance and temporary annuity contracts. The advantage of this setting is that these contracts are affected in opposite 
directions by decreased mortality: annuities cost more and life insurance costs less. In fact, if the two contracts were written on same lives, a perfect hedge for annuitant longevity would be available. In practice, there is very little mortality business in lives over 65 , however an imperfect hedge is still possible and can provide significant diversification benefits. As documented by a recent paper of Cox and Lin (2007), this "natural hedging" has a significant economic effect on the empirical annuity prices charged by US insurers. The use of term contracts is to have a well-defined optimization horizon for the insurer; this assumption can be easily modified.

In order to focus on systematic risk, we will assume that the insurer completely diversifies individual mortality risk, so that the model is stated on the level of populations. Let $0<S_{t}^{A} \leq 1$ $\left(0<S_{t}^{I} \leq 1\right)$ denote the number of all potential annuity (life insurance) holders alive at time $t$, normalized by the respective initial pools at $t=0: S_{0}^{A}=S_{0}^{I}=1$. The proportion of the cohort still present at $t$ decreases over time due to ongoing mortality events, as well as policy lapsing; the specific dynamics will be specified in the next subsection. We assume that the membership in the pool is frozen at $t=0$ so that the survivor fractions $S^{A}, S^{I}$ are non-decreasing. Let us also stress that $S^{A}$ and $S^{I}$ represent cohort pools and not only actual holders of a particular contract.

We assume that the insurer has a portfolio consisting of term life insurance policies and temporary annuities policies. Both policies have a maturity date $T$ that is fixed throughout the paper. Holders of life insurance pay continuous premiums at rate $\bar{p}_{I}$, and receive benefits of $I$ at time of death. Denoting by $\Lambda_{t}^{I}$ the corresponding force of mortality, the rate of these benefits is therefore $I \cdot \Lambda_{t}^{I} \cdot S_{t}^{I} d t$ per unit time. Conversely, annuity holders make a single upfront payment $p_{A}$, and in return receive continuous benefits at total rate $A \cdot S_{t}^{A} d t$. The constants $p_{A}, \bar{p}_{I}, A, I$ are all computed by multiplying individual premia/benefits amount by size of the group. Note that due to individual diversification, all mortality-contingent claims have continuous payment flows on $t \in(0, T)$.

\subsection{Mortality Age Structure}

The cohort of age $\{x\}$ at time $t$ experiences a mortality hazard rate $\lambda_{t}^{x}$. We assume that $\lambda^{x}$ is a stochastic process and consists of a deterministic age structure modulated by a stochastic factor $\bar{Y}^{x}$. Generically we will write $\lambda_{t}^{x} \triangleq \Lambda^{x}\left(t, \bar{Y}_{t}^{x}\right)$, and make the standing

Assumption $2.1 \Lambda:[0, T] \times \mathbb{R} \rightarrow[y, \bar{y}]$ is a positive bounded function that is twice differentiable $\Lambda \in \mathcal{C}^{2}$ and has a bounded $y$-derivative.

We will assume that $\Lambda$ also incorporates the (deterministic) lapsing rate of the cohort. Thus, for a given cohort of age $\{x\}$ at time 0 , the proportion of the cohort still present at $t$ is $S_{t}^{x} \triangleq$ $\exp \left(-\int_{0}^{t} \Lambda^{x+u}\left(u, \bar{Y}_{u}^{x+u}\right) d u\right)$. In order to make the above expression tractable, one must impose structure in some way on the collection of stochastic age-factors $\left(\bar{Y}^{x}\right)$. Three approaches seem to be possible. First, one can consider a factor model $\bar{Y}_{t}^{x}=\sum_{i=1}^{n} w^{i}(x) Y_{t}^{i}$, where $\left(Y^{1}, \ldots, Y^{n}\right)$ are $n$ stochastic factors and $w^{i}(x) \in \mathbb{R}$ are factor loadings that incorporate age-effects. For instance one may take $n=2$ and let $Y^{1} \equiv Y^{I}$ represent the young-age factor, and $Y^{2} \equiv Y^{A}$ represent the old-age factor. Then $\bar{Y}_{t}^{x}=w(x) Y_{t}^{I}+(1-w(x)) Y_{t}^{A}$ with $w(0)=0, \lim _{x \rightarrow \infty} w(x)=1$ would set the mortality factor at age $x$ as a weighted average of these two factors. Such factor models are related to Heath-Jarrow-Morton models in interest rate theory (Björk 2004), see also Schrager (2006) for a discussion in the context of stochastic mortality. 
The simplest case is a one factor model $Y^{x} \equiv Y$, meaning that the mortality age structure experiences only parallel shifts over time. Hence, the same source of uncertainty $Y$ applies to mortality rates of all ages. Thanks to its illustrative power, this setting will be explored in our first numerical example, Namely, we will take $Y$ to represent a multiplicative adjustment factor

$$
\Lambda^{x}\left(t, Y_{t}\right)=\xi(x+t) \cdot\left(Y_{t} \wedge \bar{y} \vee \underline{y}\right)
$$

with $\xi$ corresponding to a fixed mortality curve. Such a multiplicative model matches the specification proposed in Milevsky and Promislow (2001) and Dahl and Møller (2006).

Second, one may look at cohort mortality rates, so that the stochastic factor is $Y_{t}^{x}=\bar{Y}_{t}^{x+t}$ which aggregates the dynamics of $S_{t}^{x}$. More generally, given a pool $a$ of different cohorts (see next subsection), one directly postulates that the mortality rate of the pool is $\Lambda^{a}\left(t, Y_{t}^{a}\right)$ where $Y^{a}$ is unique for each pool. This is inconsistent with a mortality age-structure, but provides a straightforward mechanism for studying the dependence between mortality of different cohort pools.

Finally, one may start with a deterministic mortality age-structure and replace the parameters by stochastic processes. For example, consider

$$
\lambda_{t}^{x}=Y_{t}^{1}+Y_{t}^{2} \cdot\left(Y_{t}^{3}\right)^{x}
$$

which represents a stochastic Gompertz-Makeham curve (Dahl 2004). However, this is less parsimonious (a fully stochastic Gompertz-Makeham curve requires introduction of three stochastic factors) and does not provide a clear interpretation of $Y^{i}$ 's. Also, the induced correlation between different pools is very difficult to analyze. The one-factor model can be seen as an analogue of (2) with $Y^{3} \equiv c_{\lambda}$ taken as constant, and $Y^{1}, Y^{2}$ being perfectly correlated. Note that unlike the first two approaches, this framework does not permit separation between deterministic age-structure and stochastic drivers which is desirable on an comparative level.

\section{Age Distributions}

A realistic actuarial pool has an age distribution of insureds. This is especially true for life insurance which covers a wide selection of middle-aged cohorts. This age distribution is modeled by a probability measure $\ell_{t}$ such that the proportion of individuals in the pool between ages $x_{0}$ and $x_{1}$ at time $t$ is $\int_{x_{0}}^{x_{1}} \ell_{t}(d x)$. The future age distribution $\ell_{t+s}$ depends on the realized mortality between $t$ and $t+s$ and is given by

$$
\ell_{t+s}(s+d x)=\frac{\exp \left(-\int_{0}^{s} \Lambda^{x+u}\left(u, \bar{Y}_{u}^{x+u}\right) d u\right) \cdot \ell_{t}(d x)}{\int_{\mathbb{R}_{+}} \exp \left(-\int_{0}^{s} \Lambda^{x+u}\left(u, \bar{Y}_{u}^{x+u}\right) d u\right) \ell_{t}(d x)}
$$

In turn, the survivor fraction $S_{t}$ of the pool satisfies

$S_{t+s}=S_{t} \cdot \int_{\mathbb{R}_{+}} \exp \left(-\int_{0}^{s} \Lambda^{x+u}\left(u, \bar{Y}_{u}^{x+u}\right) d u\right) \ell_{t}(d x)=\exp \left(-\int_{0}^{s}\left[\int_{\mathbb{R}_{+}} \Lambda^{x}\left(u, \bar{Y}_{u}^{x}\right) \ell_{t+u}(d x)\right] d u\right)$.

We conclude that under stochastic mortality, the age-distribution $\ell_{t}$ is stochastic and in turn drives the evolution of the stochastic survivor fraction $S_{t}$. Moreover, even when there is a single stochastic mortality factor $Y$, the nonlinearities above imply that there is no sufficient finitedimensional statistic to describe the evolution of $\ell_{t}$. Hence, the evolution of age-composition of 
the entire pool (and therefore also the evolution of its overall mortality rate) is path-dependent and non-Markovian unless one counts the infinite-dimensional $\ell_{t}$ as a state variable. This makes direct analysis intractable and instead requires approximations.

For annuity pools, explicit modeling of stochastic $S_{t}^{A}$ is absolutely necessary since the cashflows are given by $A \cdot S_{t}^{A}$ and lapsing is minimal because the annuitants pay a single upfront premium. At the same time, the age variability in new annuitants is minimal (as we consider a one-time sale of contracts), so a reasonable approximation is to take all annuitants to be of same initial age $\left\{x^{A}\right\}$ and set

$$
S_{t}^{A}=\exp \left(-\int_{0}^{t} \Lambda^{x^{A}+u}\left(u, \bar{Y}_{u}^{x^{A}+u}\right) d u\right)
$$

After that the $\left\{\bar{Y}^{x^{A}+u}\right\}$ can be aggregated using any of the approaches mentioned before.

On the other hand, life insurance pools experience high rates of policy lapsing which typically dominate the relatively low mortality rates. Consequently, on a practical level the stochastic changes in insurance pool composition and survivor fraction due to systematic mortality risk are averaged out by concurrent policy lapses. Thus, a reasonable assumption is that $S_{t}^{I}$ and corresponding $\ell_{t}^{I}$ are deterministic and the dynamics of the insurance survivor fraction $S^{I}$ are

$$
S_{t}^{I}=\exp \left(-\int_{0}^{t} \int_{\mathbb{R}_{+}} \Lambda^{x}(u) \ell_{u}^{I}(d x) d u\right),
$$

where $\Lambda^{x}(u)$ denotes a deterministic lapsing rate of the $\{x\}$-cohort at time $u$. Note that the insurance cashflow from pool mortality is still stochastic $\int_{\mathbb{R}_{+}} \Lambda^{x}\left(t, \bar{Y}_{t}^{x}\right) \ell_{t}^{I}(d x) \cdot S_{t}^{I}$.

In the case of a single mortality factor $Y$, an alternative is to assume that the relative ratio of $S^{A}$ and $S^{I}$ is deterministic, $S_{t}^{I}=p_{t} S_{t}^{A}$ where $p_{t}$ is the deterministic proportionality constant. This fixed relative size of the two pools is assumed due to ongoing policy lapses that obscure the increased mortality of old ages. The approach can be taken further: one may consider a single "global" pool $S$ with deterministic age distribution $\ell_{t}$. The shrinkage rate of the pool is stochastic and given by $\Lambda\left(t, Y_{t}\right)$. Finally, the pool's $\{x\}$-cohort at date $t$ is divided into a deterministic proportion $\ell_{t}^{I}(d x)$ of potential life insurance holders and another proportion $\ell_{t}^{A}(d x)$ who are potential annuity holders. Again, this can be justified by lapsing rates dominating the stochastic fluctuations.

\section{Mortality Dynamics}

An ongoing debate exists regarding appropriate models for time-evolution of the mortality factor(s) $Y$. Since our model is in continuous-time, we postulate that each $Y$ is of a linear diffusion type, so that

$$
d Y_{t}=\mu\left(Y_{t}\right) d t+\sigma d W_{t}^{y}, \quad Y_{0}=y,
$$

where $W^{y}$ is a Brownian motion on a stochastic basis $(\Omega, \mathcal{F}, \mathbb{P})$. We make the following standing technical assumptions regarding (4):

Assumption $2.2 \mu(y)=\theta_{Y}-\kappa y$ is a linear function and $\sigma$ is a positive constant (we can take $\sigma$ to be a positive function of $y$ which is bounded from above, and below away from zero).

Because the actual mortality rate is of the form $\Lambda\left(t, Y_{t}\right)$, there is little loss of generality in making the above assumptions since many additional transformations of $Y$ can be incorporated into $\Lambda$. 
Let us note that an alternative class of discrete-time models has been used in the literature, see e.g. Lee and Carter (1992), Cairns et al. (2004). However, since in our dynamic setup the insurer hedges continuously, it would be unnatural to assume that mortality rates change discretely (the latter would cause discontinuities in hedging strategies). See Ludkovski and Young (2007) for a discussion of a mixed continuous-time/discrete-time stochastic mortality model.

\subsection{Interest Rate Model and Bond Market}

Because annuities pay money over a long period of time, annuity writers are exposed to significant inflation and interest rate risk. In fact, the latter is the major source of uncertainty in payouts made. To hedge interest rate risk, insurers invest in long-term (government) bonds that remove as much as possible future reinvestment risk. In order to appropriately model the corresponding fluctuations, we will explicitly model interest rates in a simple single-factor stochastic spot rate model. As explained in Section 4.2, the assumption of stochastic force of interest is crucial for obtaining reasonable results. We assume that the insurer invests in a risk-free bank account yielding the force of interest $r_{t}$ at time $t$, and also trades in long-term bonds of maturity $T_{1}>T$, priced at $F\left(r_{t}, t ; T_{1}\right)$. Since the considered contracts do not include equity risk, we exclude possibility of trading in stocks.

We take the following Affine Term Structure (see Björk (2004)) model for the short interest rate $r_{t}$ :

$$
d r_{t}=\left(a_{0}(t) r_{t}+b_{0}(t)\right) d t+\sqrt{c(t) r_{t}+d(t)} d W_{t}^{r}
$$

where $W^{r}$ is another Brownian motion independent of the Brownian motion(s) $W^{y}$, and $a_{0}, b_{0}, c, d$ are deterministic functions. The framework of (5) includes the commonly used Vasicek, Ho-Lee and Cox-Ingersoll-Ross models. We will assume that the market prices the $T_{1}$-bonds under the probability measure $\mathbb{Q}$ whose Radon-Nikodym derivative with respect to $\mathbb{P}$ is

$$
\frac{d \mathbb{Q}}{d \mathbb{P}}=\exp \left(-\int_{0}^{T_{1}} \frac{\left(a_{0}-a\right) r_{t}+\left(b_{0}-b\right)}{\sqrt{c r_{t}+d}} d B_{t}-\frac{1}{2} \int_{0}^{T_{1}} \frac{\left(\left(a_{0}-a\right) r_{t}+\left(b_{0}-b\right)\right)^{2}}{c r_{t}+d} d t\right) .
$$

We write $\Delta a=\left(a_{0}-a\right), \Delta b=\left(b_{0}-b\right)$ and recall that $\widetilde{W}_{t}^{r}=W_{t}^{r}+\int_{0}^{t}\left(\Delta a r_{s}+\Delta b\right) / \sqrt{c r_{s}+d} d t$ defines a $\mathbb{Q}$-Brownian motion. The latter term is interpreted as the market price of interest rate risk. We also recall that the $T_{1}$-bond price $F\left(r, t ; T_{1}\right)=\mathbb{E}^{\mathbb{Q}}\left[\mathrm{e}^{-\int_{t}^{T_{1}} r_{s} d s} \mid r_{t}=r\right]$ is a $\mathbb{Q}$-martingale and is given by (see Björk (2004)),

$$
\left\{\begin{array}{l}
F\left(r, t ; T_{1}\right)=\mathrm{e}^{A\left(t, T_{1}\right)-C\left(t, T_{1}\right) r} ; \\
C_{t}\left(t, T_{1}\right)+a(t) C\left(t, T_{1}\right)-\frac{1}{2} c(t) C\left(t, T_{1}\right)^{2}=-1, \quad C\left(T_{1}, T_{1}\right)=0, \\
A_{t}\left(t, T_{1}\right)=b(t) C\left(t, T_{1}\right)-\frac{1}{2} d(t) C\left(t, T_{1}\right)^{2}, \quad A\left(T_{1}, T_{1}\right)=0,
\end{array}\right.
$$

where subscripts denote derivatives with respect to corresponding variables. Also note that the bond sensitivity to interest rates satisfies $F_{r}=-C\left(t, T_{1}\right) F, F_{r r}=C\left(t, T_{1}\right)^{2} F$. 


\subsection{Dynamic Exponential Premium Principle}

Actuaries typically price insurance products using a premium rule. Thus, given a premium principle $U$, the price charged for a contract which pays out $f_{1}$ at time $T$ is $\$ H$ such that

$$
x=\mathbb{E}\left[U\left(x-f_{1}+H\right)\right],
$$

where $x$ is the initial endowment of the insurer (Bowers et al. 1997). This is a static view of the problem; in a dynamic setting the insurer also makes intermediate trades that affect her terminal endowment so that the model incorporates optimization, or more formally an optimal investment problem. To be consistent, optimal investment should be considered on both sides of (8). Furthermore, if besides $f_{1}$ the insurer also sold $f_{2}$, then the relative premium rule pricing $H^{f_{1} \mid f_{2}}$ becomes

$$
\sup _{\pi} \mathbb{E}\left[U\left(X^{x, \pi}-f_{1}-f_{2}+H^{f_{1} \mid f_{2}}\right)\right]=: \sup _{\pi} \mathbb{E}\left[U\left(X^{x, \pi}-f_{2}\right)\right],
$$

where $X^{x, \pi}$ denotes the endowment process of the insurer starting with initial capital $x$ and making trades according to $\pi$. This leads to the so-called relative indifference pricing mechanism. In plain words, the relative indifference price of a mortality contingent contract $f_{1}$ given another contract $f_{2}$ is defined to be the amount $H^{f_{1} \mid f_{2}}$ dollars such that the insurer derives the same indirect utility from selling $f_{1}$ and $f_{2}$ and receiving $H^{f_{1} \mid f_{2}}$ dollars, or alternatively only selling $f_{2}$. For brevity, we will write $H^{f} \equiv H^{f \mid 0}$, and just $H$ whenever the claim $f$ considered is clearly understood. By definition, $H^{f_{1} \mid f_{2}} \triangleq H^{f_{1}+f_{2}}-H^{f_{2}}$ and due to the non-linear pricing structure, $H^{f_{1} \mid f_{2}} \neq H^{f_{1}}$, see Corollary 3.1. In terms of parameters, $H^{f_{1} \mid f_{2}}$ is a function of the current interest rates, current mortality rates, current number of insureds and the insurer's wealth.

In this paper we use the exponential premium principle $U(x)=-\exp (-\gamma x)$. This principle is well-known in the actuarial literature as the Esscher transform. One of its most attractive features is wealth-invariance: the resulting premium $H$ is independent of $x$. This is useful since it makes pricing independent of the capital structure of the insurer which is a reasonable assumption for a large insurance writer. Secondly, the exponential premium principle possesses nice analytical properties that simplify the mathematical analysis. In that sense, it is a good representative of indifference-pricing mechanism due to its tractability. Finally, the exponential principle is very simple and only has one parameter $\gamma$ which can be used to summarize the risk-attitudes of the insurer. As shown by Gerber (1976), in a static setting $\gamma$ can be related to the probability of ruin and thus may be roughly estimated once we know the insurer's reserves. In Section 4.1 we compare this choice to another premium principle based on good deal bounds.

\subsection{Case Study}

In our primary case-study we assume that the insurer has an established life insurance business and is planning on starting an annuity line. Specifically, the insurer already sold life insurance contracts to a given fraction $\beta \leq 1$ of the $S^{I}$-pool and is considering a one-time sale of annuities to a fraction $\alpha \leq 1$ of the $S^{A}$-pool. If she does so, the insurer's cashflow rate at time $t$ would be

$$
-f d t \triangleq\left(-\alpha \cdot A \cdot S_{t}^{A}-\beta \cdot I \cdot \Lambda^{I}\left(t, Y_{t}\right) \cdot S_{t}^{I}+\beta \cdot \bar{p}_{I} \cdot S_{t}^{I}\right) d t,
$$

and our main object of interest will be the relative indifference price

$$
H^{\alpha A \mid \beta I}=H^{\alpha A+\beta I}-H^{\beta I} \text {. }
$$


$H^{\alpha A \mid \beta I}$ captures the relative hedging since it involves optimization over the combined cashflows with respect to the non-linear risk-preferences. $H^{\alpha A \mid \beta I}$ should be thought of as the internal cost to the insurer of selling $\alpha$ annuities, given its existing portfolio of $\beta$ life insurances. The converse situation of an annuities insurer entering life-insurance business is discussed in Remark 5.1. Pricing of more exotic insurance contracts, such as certain-and-life or deferred annuities, varying benefit insurance and semi-continuous products can also be accommodated. Let us stress that (11) reflects a "one-time" sale of annuities — next period (say next year), the problem must be redone since $H$ depends on the insurer's portfolio which itself changes over time. Similar valuation problems have been explored in Bayraktar and Young (2007a) using the instantaneous Sharpe ratio premium principle (see Section 4.1) and in Cox and Lin (2007) using the static Wang transform method.

As we will see, $H^{\alpha A \mid \beta I}$ is strictly increasing and convex in $\alpha$. On the other hand the proceeds from market sales $p_{\alpha A}=\alpha A p$ are linear in quantity. Consequently, it is meaningless to talk about $H^{\alpha A \mid \beta I}$ per say; instead we formulate an optimization problem in terms of finding the optimal quantity $\alpha$ for an insurer to sell in a competitive annuity market. Thus, we assume that the annuity upfront premium is set exogenously at $p_{A}$. The insurer would like to maximize its expected gain by choosing the quantity $\alpha$ of annuities to sell, given its portfolio and this prevailing market price. In terms of our notation, the insurer seeks

$$
\alpha^{*}=\operatorname{argmax}_{\alpha}\left(\alpha \cdot p_{A}-H^{\alpha A \mid \beta I}\right) .
$$

An alternative formulation for a monopoly insurer is discussed in Remark 6.4.

\section{$3 \quad$ Single Pool Model}

In this section we consider a single pool $S$ and a single stochastic factor $Y$. This case can be interpreted as a homogeneous population consisting of a single cohort of same age $\{x\}$, or as a global pool with a deterministic ratio $p_{t}$ between the annuity and insurance subgroups. In both cases the age-effects in the pool's age distribution are absorbed into the time-dependency of $\Lambda\left(t, Y_{t}\right)$ and the dynamics of the pool survivor fraction are simply

$$
d S_{t}=-\left[\Lambda\left(t, Y_{t}\right)\right] S_{t} d t
$$

In the latter interpretation, all the extra constants are absorbed into the cashflow rates $\bar{p}_{I}, I, A$. All the extra constants are absorbed into the cashflow rates $\bar{p}_{I}, I, A$, for example we can take $\Lambda^{I}\left(t, Y_{t}\right)=c \Lambda\left(t, Y_{y}\right)=\Lambda^{A}\left(t, Y_{t}\right)$ for some $c \in(0,1)$, but we can absorb this constant into $c$.

\subsection{Utility Indifference Price}

To derive the dynamic exponential premium $H^{f}$ of a mortality contingent claim $f$, we must first consider optimal insurer investment strategy with and without $f$. This programme is briefly carried out in this section; proofs of the theorems are given in the Appendix.

\section{Utility Maximization without Contingent Claims}

Without any claims, the insurer invests in the $T_{1}$-bonds and the bank account. Let $\pi_{t}$ be the dollar amount invested in the $T_{1}$-bonds at time $t$ and denote by $X_{t}$ the wealth accumulated by 
date $t$, with $w$ the initial endowment. Then

$$
\begin{aligned}
d X_{t} & =r_{t}\left(X_{t}-\pi_{t}\right) d t+\pi_{t} d F_{t} / F_{t} \\
& =\left[r_{t} X_{t}-C\left(t, T_{1}\right)\left(\Delta a r_{t}+\Delta b\right) \pi_{t}\right] d t-C\left(t, T_{1}\right) \sqrt{c r_{t}+d} \pi_{t} d W_{t}^{r} \quad X_{0}=x .
\end{aligned}
$$

We will denote by $\mathcal{A}$ the set of admissible trading strategies $\pi$ given by

$$
\mathcal{A} \triangleq\left\{\pi: \mathcal{F} \text { - measurable, } \mathbb{P}\left[\int_{0}^{T}\left(c r_{t}+d\right) \pi_{t}^{2} d t<\infty\right]=1\right\} .
$$

The value function for maximizing terminal utility is defined as

$$
V(x, r, t) \triangleq \sup _{\pi \in \mathcal{A}} \mathbb{E}^{x, r, t}\left[-\mathrm{e}^{-\gamma X_{T}}\right]
$$

where $\gamma>0$ is the risk-aversion coefficient and $\mathbb{E}^{x, r, t}$ denotes the conditional expectation $\mathbb{E}\left[\cdot \mid X_{t}=\right.$ $\left.x, r_{t}=r\right]$. Note that the problem is stated under the physical measure $\mathbb{P}$. Thus, $V$ measures the maximum terminal utility the agent can achieve by time $T$ using an admissible trading strategy.

Theorem 3.1 We have (Ludkovski and Young 2007)

$$
V(x, r, t)=-\exp \left(-\frac{\gamma}{F(r, t ; T)} x+\psi(r, t)\right),
$$

where $\psi$ is the unique smooth solution of the linear pde

$$
\left\{\begin{array}{l}
\psi_{t}+[(a r+b)-C(t, T)(c r+d)] \psi_{r}+\frac{1}{2}(c r+d) \psi_{r r} \\
\quad-\frac{1}{(c r+d)}[(\Delta a r+\Delta b)+C(t, T)(c r+d)]^{2}=0, \\
\psi(r, T)=0 .
\end{array}\right.
$$

The term $F^{-1}(r, t ; T)$ in (13) represents the discount factor between time $t$ and $T$, and the second term $\psi(r, t)$ corresponds to extra profit available thanks to trading opportunities. In terms of certainty equivalents, $x$ dollars at time $T$ are worth $x / F(r, t ; T)-\psi(r, t) / \gamma$ dollars to the agent today.

\section{Utility with Contingent Claims}

Consider a mortality contingent claim that pays at a continuous rate $f\left(\Lambda\left(t, Y_{t}\right), S_{t}\right)$ per unit time. The wealth of the insurer then satisfies

$$
d X_{t}^{f}=\left\{r_{t} X_{t}^{f}-C\left(t, T_{1}\right)\left(\Delta a r_{t}+\Delta b\right) \pi_{t}\right\} d t-C\left(t, T_{1}\right) \sqrt{c r_{t}+d} \pi_{t} d W_{t}^{r}-f\left(\Lambda\left(t, Y_{t}\right), S_{t}\right) d t .
$$

We again look at the problem of maximizing utility of terminal wealth

$$
U^{f}(x, y, r, S, t) \triangleq \sup _{\pi \in \mathcal{A}} \mathbb{E}^{x, \vec{z}, t}\left[-\mathrm{e}^{-\gamma X_{T}^{f}}\right] \quad \text { with } \vec{z}=(y, r, S) .
$$

Define the second-order differential operator

$$
\mathcal{L}_{H} \triangleq(a r+b) \partial_{r}+\frac{1}{2}(c r+d) \partial_{r r}^{2}+\mu(y) \partial_{y}+\frac{1}{2} \sigma^{2} \partial_{y y}^{2}-\Lambda(t, y) S \partial_{S} .
$$


Theorem 3.2 We have

$$
U^{f}(x, y, r, S, t)=V(x, r, t) \mathrm{e}^{\gamma L(y, r, S, t)},
$$

where $L$ satisfies

$$
\left\{\begin{aligned}
L_{t}+\mathcal{L}_{H} L-C\left(t, T_{1}\right) L_{r}+\frac{1}{2} \sigma^{2} \gamma L_{y}^{2}+\frac{f\left(\Lambda\left(t, Y_{t}\right), S_{t}\right)}{F(r, t ; T)} & =0 \\
L(y, r, S, T) & =0
\end{aligned}\right.
$$

\section{Representations for Exponential Premium}

Recall that the exponential premium $H^{f}$ of claim $f$ is defined via

$$
V(x, r, t)=U^{f}\left(x+H^{f}, y, r, S, t\right) .
$$

$H^{f}$ can be interpreted as the reserve requirement for $f$ : at time $t$, the insurer is indifferent between holding on to $f$ or paying $H^{f}(y, r, S, t)$ to a re-insurer to take on the corresponding risks.

Comparing with (17) implies that $H^{f}$ is independent of endowment $x$ and is equal to

$$
H^{f}(y, r, S, t)=F(r, t ; T) \cdot L(y, r, S, t) .
$$

Note that the premium does not depend on the maturity of the bond that the insurer uses to hedge the interest rate risk. This is because the bond market is complete, and hence any given bond with maturity $T^{\prime}$ can be perfectly replicated by a self-financing strategy in the $T_{1}$-bond. Thus, the reinvestment risk is zero.

Theorem 3.3 Let

$$
K(p) \triangleq \frac{1}{2} \sigma^{2} \frac{\gamma}{F(r, t ; T)} p^{2}
$$

The exponential premium $H$ is the unique classical solution of

$$
H_{t}+\mathcal{L}_{H} H+K\left(H_{y}\right)-r H+f(\Lambda(t, y), S)=0, \quad H(y, r, S, T)=0,
$$

satisfying polynomial growth conditions. Moreover, $H$ has the stochastic representation

$H(y, r, S, t)=\sup _{\left(q_{t}\right)_{t \geq 0}} \mathbb{E}^{Q^{q}}\left[\int_{t}^{T} \mathrm{e}^{-\int_{t}^{u} r_{s} d s}\left(-q_{u}^{2} \frac{F\left(r_{u}, u ; T\right)}{2 \gamma \sigma^{2}}+f\left(\Lambda\left(u, Y_{u}\right), S_{u}\right)\right) d u \mid Y_{t}=y, r_{t}=r, S_{t}=S\right]$,

in which the controlled dynamics of $Y$ under the measure $Q^{q} \simeq \mathbb{P}$ are given by

$$
d Y_{t}=\left(\mu\left(Y_{t}\right)+q_{t}\right) d t+\sigma d W_{t}^{q}, \quad Y_{0}=y,
$$

with $W^{q}$ a $Q^{q}$-Brownian motion and the dynamics of $r$ are same as under $\mathbb{Q}$.

We see that (20) is a semi-linear pde that resembles the typical Black-Scholes pde for a contingent claim with continuous payments at rate $f$ (see e.g. Björk (2004)), except for the nonlinear term $K\left(H_{y}\right)$ which takes into account the systematic mortality risk and the trading opportunities in the bond.

The stochastic representation (21) provides us immediately with the following several corollaries that show the basic properties of the exponential premium pricing mechanism. 
Corollary 3.1 Indifference prices are super-additive: $H^{X+Y} \geq H^{X}+H^{Y}$ for any contingent claims $X, Y$.

Corollary 3.2 Indifference prices are convex in quantities, i.e., as a function of $\alpha, H$ is convex.

This follows because for $H^{\alpha X}$ the expectation inside the sup in (21) is linear in $\alpha$; a supremum of linear functions is convex.

Corollary 3.3 Indifference prices increase in the risk parameter $\gamma$.

This is because in (21), as $\gamma$ increases, the denominator of the first (negative) term increases, increasing the expectation for any fixed $\left(q_{t}\right)$.

Theorem 3.4 For any cashflow rate $f$, we have that $\operatorname{sgn}\left(H_{r}\right)=-\operatorname{sgn}\left(F_{r}\right) \geq 0, \operatorname{sgn}\left(H_{S}\right)=$ $\operatorname{sgn}\left(f_{S}\right)$. If we assume that the function $\lambda \rightarrow f(\lambda, t)$ is non-decreasing with respect to its first variable and $y \rightarrow \Lambda(t, y)$, is increasing in $y$ for all $t \in[0, T]$, then $H_{y} \geq 0$. If we additionally assume that $y \rightarrow \Lambda(t, y)$ and $\lambda \rightarrow f(\lambda, t)$ are convex for $t \in[0, T]$, then $H_{y y} \geq 0$.

The theorem shows that higher interest rates always decrease required reserves. The increase in the mortality rate has the opposite effect.

Theorem 3.5 Assume that $\Lambda(t, y)$ in (21) is an increasing and convex function of its second variable. Then if either of $\mu$ or $\sigma$ in (20) increases, the corresponding indifference prices increase as well.

Note that the assumption in the theorem is satisfied in all the approaches of Section 2.1. Thus, we see that a more volatile mortality rate (and hence a stronger exposure to systematic mortality risk) increases the exponential premia.

\section{$3.2 \quad$ Indifference Hedging Strategy}

Theorem 3.6 The optimal hedging strategy of claim $f$ is given in feedback form as

$$
\pi_{t}^{f}=\frac{1}{C\left(t, T_{1}\right)}\left[C(t, T)\left(X_{t}^{f}-X_{t}^{0}-H(\cdot, t)\right)-H_{r}(\cdot, t)\right] .
$$

The difference $N_{t} \triangleq X_{t}^{f}-H\left(Y_{t}, r_{t}, S_{t}, t\right)$ can be interpreted as the net worth of the firm, since it is the nominal wealth at time $t$ minus the current expected liability of $H\left(Y_{t}, r_{t}, S_{t}, t\right)$. The term $H_{r}\left(Y_{t}, r_{t}, S_{t}, t\right)$ corresponds to the Delta of Black-Scholes dynamic trading model and accounts for hedging in the (financially complete) interest-rate market. A similar expression was obtained in Young (2003). It can be shown that if $f$ contains no systematic risk (e.g. no dependence on $\Lambda$ ) then $\pi_{t}^{f}$ will equal the unique replicating portfolio of $f$. This is one of the special properties of exponential premium principle. 
Applying Ito's formula we can compute the time-evolution of the indifference value $H \equiv$ $H\left(Y_{t}, r_{t}, S_{t}, t\right)$ as

$$
d H_{t}=\left[r_{t} H+(\Delta a r+\Delta b) H_{r}-\frac{1}{2} \sigma^{2} \frac{\gamma}{F\left(r_{t}, t ; T\right)}\left(H_{y}\right)^{2}-f\right] d t+H_{r} \sqrt{c r_{t}+d} d W_{t}^{r}+H_{y} \sigma d W_{t}^{y} .
$$

Combining with (15) and $(23)$ and setting $\Phi=\left[-\frac{\Delta a r_{t}+\Delta b}{c r_{t}+d}-C(t, T)-\psi_{r}(\cdot, t)\right]$ we obtain the dynamics of the net worth $N_{t}$ of the firm,

$$
\begin{array}{r}
d N_{t}=\left\{r_{t} N+\left(\Delta a r_{t}+\Delta b\right) C(t, T) N+\frac{1}{2} \sigma^{2} \frac{\gamma}{F\left(r_{t}, t ; T\right)}\left(H_{y}\right)^{2}+\frac{F\left(r_{t}, t ; T\right)}{\gamma}\left(\Delta a r_{t}+\Delta b\right) \Phi\right\} d t \\
-\sqrt{c r_{t}+d}\left\{C(t, T) N+\frac{F\left(r_{t}, t ; T\right)}{\gamma} \Phi\right\} d W_{t}^{r}-\sigma H_{y} d W_{t}^{y} .
\end{array}
$$

\subsection{Bounds on the Indifference Price}

Before proceeding with investigation of the semi-linear pde (A.59), we provide next some initial bounds on its solution.

Theorem 3.7 Let $G \triangleq \int_{t}^{T} \frac{f\left(\Lambda\left(u, Y_{u}\right), S_{u}\right)}{F\left(r_{u}, u ; T\right)} d u$ be the forward cumulative value of the cashflows corresponding to claim $f$. Then the exponential premium $H^{f}$ of $f$ satisfies the bounds

$$
F(r, t ; T) \mathbb{E}_{t}^{\mathbb{Q}}[G] \leq H^{f}(y, r, S, t) \leq \frac{F(r, t ; T)}{\gamma} \ln \left(\mathbb{E}_{t}^{\mathbb{Q}}\left[\mathrm{e}^{\gamma G}\right]\right)
$$

Observe that the lower bound is linear in the forward cumulative benefit $G$, and is related to the standard risk-neutral pricing mechanism. In particular, the lower bound is independent of $\gamma$. On the other hand, the upper bound is nonlinear and is a function of $\gamma$; it can be recognized as the Esscher transform (Bowers et al. 1997) of $G$. As $\gamma \rightarrow \infty$, the upper bound converges to the super-hedging price of $G$; for instance for a $T$-year temporary life annuity paying out $A$ per year, the super-hedging price is simply $T \cdot A$.

\section{Other Pricing Mechanisms}

\subsection{Instantaneous Sharpe Ratio Premium Principle}

In this section we will obtain the cost, denoted by $\mathcal{H}$, of delivering $f$ instantaneously in the pricing framework of Bayraktar and Young (2008), Young (2008) and Bayraktar and Young (2007a). This framework assumes that the insurer requires compensation for the mortality risk it assumes in the form of a pre-specified Sharpe ratio, which is a continuous version of the standard-deviation premium principle.

Theorem 4.1 Let $\gamma^{S R} \geq 0$ denote the instantaneous Sharpe ratio required by the insurer. Then the resulting indifference price $\mathcal{H}^{f}$ solves

$$
\mathcal{H}_{t}+\mathcal{L}_{H} \mathcal{H}-r \mathcal{H}+f(\Lambda(t, y), S)=-\gamma^{S R} \sigma\left|\mathcal{H}_{y}\right|, \quad \mathcal{H}(y, r, S, T)=0 .
$$


Moreover, $\mathcal{H}$ has the stochastic representation,

$$
\mathcal{H}(y, r, S, t)=\sup _{\left(q_{t}\right)_{t \geq 0}\left|q_{t}\right| \leq \gamma^{S R_{\sigma}}} \mathbb{E}^{Q^{q}}\left[\int_{t}^{T} \mathrm{e}^{-\int_{t}^{u} r_{s} d s} f\left(\Lambda\left(u, Y_{u}\right), S_{u}\right) d u \mid Y_{t}=y, r_{t}=r, S_{t}=S\right]
$$

where the measure $Q^{q}$ is as in (21).

The representation of $\mathcal{H}$ indicates that $\mathcal{H}$ is the upper good deal bound in the sense of robust optimization over a set of martingale measures Björk and Slinko (2006). This correspondence between the standard deviation premium principle using the instantaneous Sharpe ratio and the good deal bounds was observed in Bayraktar and Young (2008). In particular, when $\gamma^{S R}=0$, $\mathcal{H}$ is equal to the expectation under the minimal martingale measure $\mathbb{Q}$ and is given by the right-hand-side of (40).

Remark 4.1 As a result of the representation in (28), we have that $\mathcal{H}$ is increasing in $\gamma$ and that it is convex in $\alpha$.

Remark 4.2 If $f=f^{\alpha A+\beta I}$ satisfies $f_{y}>0$ (in our case study this is always the case), and $f_{S}<0$ (in our case study this is satisfied when e.g. $\beta \bar{p}_{I}>\alpha A+\beta I \bar{y}$ ) then $\mathcal{H}_{y}>0$ (the proof of this result is similar to that of Theorem 2.3 in Bayraktar and Young (2008)). On the other hand, if $f_{y}=0$ and $f_{S}<0$ (the pure annuities case), then $\mathcal{H}_{y}<0$. Consequently, the nonlinear absolute value operator in (27) can be dropped and the resulting linear equation has the Feynman-Kac representation (Karatzas and Shreve 1991)

$$
\mathcal{H}(y, r, S, t)=\mathbb{E}^{ \pm Q^{S R}}\left[\int_{t}^{T} f\left(\Lambda\left(u, Y_{u}\right), S_{u}\right) \mathrm{e}^{-\int_{t}^{u} r_{s} d s} d u \mid Y_{t}=y, r_{t}=r, S_{t}=S\right]
$$

where under $Q^{ \pm S R} \equiv Q^{ \pm \gamma^{S R} \sigma}$, the mortality factor solves $d Y_{t}=\left(\mu\left(Y_{t}\right) \pm \gamma^{S R} \sigma\right) d t+\sigma d W_{t}^{q}$. In other words the premium principle distorts the dynamics of $Y$ via a Girsanov transformation to make mortality more/less likely which in turn respectively increases value of life insurance/increases the value of annuities.

Note that when $f_{y}>0$ and $f_{S}<0$, then according to the price scheme in this section, there is no cross hedging: $\mathcal{H}^{\alpha A+\beta I \mid \beta I}=\mathcal{H}^{\alpha A}$. But when $f_{y}>0$ and $f_{S}>0$, then it is hard to say anything definitive about the sign of $\mathcal{H}_{y}$ and the pde that $\mathcal{H}$ solves is not linear. In this case one has cross hedging: $\mathcal{H}^{\alpha A+\beta I \mid \beta I} \leq \mathcal{H}^{\alpha A}$ (this inequality immediately follows from (28)).

\subsection{Investing in Stock Market}

In our model we have deliberately ignored investment in a stock market. This is because we assume that the mortality risk is independent of other economic variables. This assumption implies that if a Black-Scholes model is used for the stock dynamics, the resulting indifference premium is trivial. To elaborate, consider a stock market evolving according to

$$
d P_{t}=\mu P_{t} d t+\sigma P_{t} d W_{t}^{P}
$$

where $W^{P}$ is another independent Brownian motion. To isolate the effect of stock investments we take constant interest rates $r_{t}=r$, so that $F(r, t ; T)=\mathrm{e}^{-r(T-t)}$. The insurer invests $\pi_{t}$ 
dollars into the stock market at time $t$ and has a portfolio of insurance claims with cashflows $f$. Her wealth $X$ then satisfies

$$
d X_{t}^{f}=\left\{r X_{t}^{f}+\mu \pi_{t}\right\} d t+\sigma \pi_{t} d W_{t}^{P}-f d t
$$

Solving for $V(x, p, y, t)=\sup _{\pi \in \mathcal{A}} \mathbb{E}_{t}^{x, p, y}\left[-\mathrm{e}^{\left.-\gamma X_{T}^{x, \pi}\right]}\right.$ we find (Henderson 2005)

$$
V(x, p, y, t)=-\mathrm{e}^{-\gamma \mathrm{e}^{r(T-t)} x} \cdot \mathrm{e}^{-(\mu-r)^{2} / 2 \sigma^{2}(T-t)} \cdot \mathbb{E}_{t}^{\mathbb{Q}}\left[\exp \left(-\gamma \int_{t}^{T} \mathrm{e}^{r(T-u)} f\left(\Lambda\left(u, Y_{u}\right), S_{u}\right) d u\right)\right] .
$$

Hence the indifference price is

$$
H(y, t)=\frac{\mathrm{e}^{-r(T-t)}}{\gamma} \ln \mathbb{E}^{\mathbb{Q}}\left[\mathrm{e}^{\gamma \int_{t}^{T} \mathrm{e}^{r(T-u)} f\left(\Lambda\left(u, Y_{u}\right), S_{u}\right) d u}\right]=\frac{F(r, t ; T)}{\gamma} \ln \mathbb{E}^{\mathbb{Q}}\left[\mathrm{e}^{\gamma G}\right],
$$

which we recognize as the upper bound in (26). Moreover, the optimal strategy is $\pi^{f} \equiv 0$ not to hedge $f$ at all. This is the expected behavior of an optimizer in an incomplete market given a totally unhedgeable risk - the insurer just ignores the uncertainty and proceeds in a trivial way (Becherer 2003). In light of these negative results, we consider the harder problem of a risky bond market which allows hedging the interest rate exposure of the insurer.

A realistic insurer does invest in the stock market, but for reasons beyond our model. These will include: attempt to achieve returns above the available risk-free rate; hedging against equitylinked insurance products; hedging against mortality risk by investing in shares of mortalityrelated businesses (such as pharmaceutical companies, nursing care industry, etc.). However, such investments usually carry a lot of company-specific risk and are arguably a very inefficient way of mitigating systematic mortality exposure. It would be interesting to consider such possibilities in future work.

\section{$5 \quad$ Framework for Numerical Examples}

To illustrate our results we consider the mortality model of Dahl and Møller (2006). We model the mortality rate as $\lambda_{t}=\Lambda\left(t, Y_{t}\right)=\xi\left(t+x^{0}\right) \cdot\left(Y_{t} \wedge \bar{y} \vee y\right)$ where $\xi\left(t+x^{0}\right)$ is the deterministic mortality curve fitted to current data, and $Y_{t}$ is the mortality adjustment factor. The reference pool consists initially of $10^{6}$ individuals, all of age $x^{0}=65$. We take the Gompertz-Makeham mortality curve

$$
\xi(z)=a_{\lambda}+b_{\lambda} \cdot\left(c_{\lambda}\right)^{z},
$$

in which the parameters $a_{\lambda}, b_{\lambda}$ and $c_{\lambda}$ are fitted to US mortality data from 2000 as reported by Dahl and Møller (2006). Figure 1 shows the resulting mortality age structure for ages 60-85, which is also very close to the 1990-95 US SOA Basic Life Insurance Table. We take $T=20$-year contracts, so that under the deterministic mortality curve, ${ }_{20} p_{65}=30.08 \%$ of the population is expected to survive until age 85 and receive full annuity benefits.

For the mortality adjustment factor $Y$ we assume an Ornstein-Uhlenbeck process with mean level one:

$$
d Y_{t}=\kappa_{\lambda}\left(1-Y_{t}\right) d t+\sigma_{\lambda} d W_{t} .
$$

Recall that $Y$ is a multiplicative adjustment factor, so that $Y>1$ means that mortality is higher than expected. Expected mortality improvements can be built-in by incorporating a 


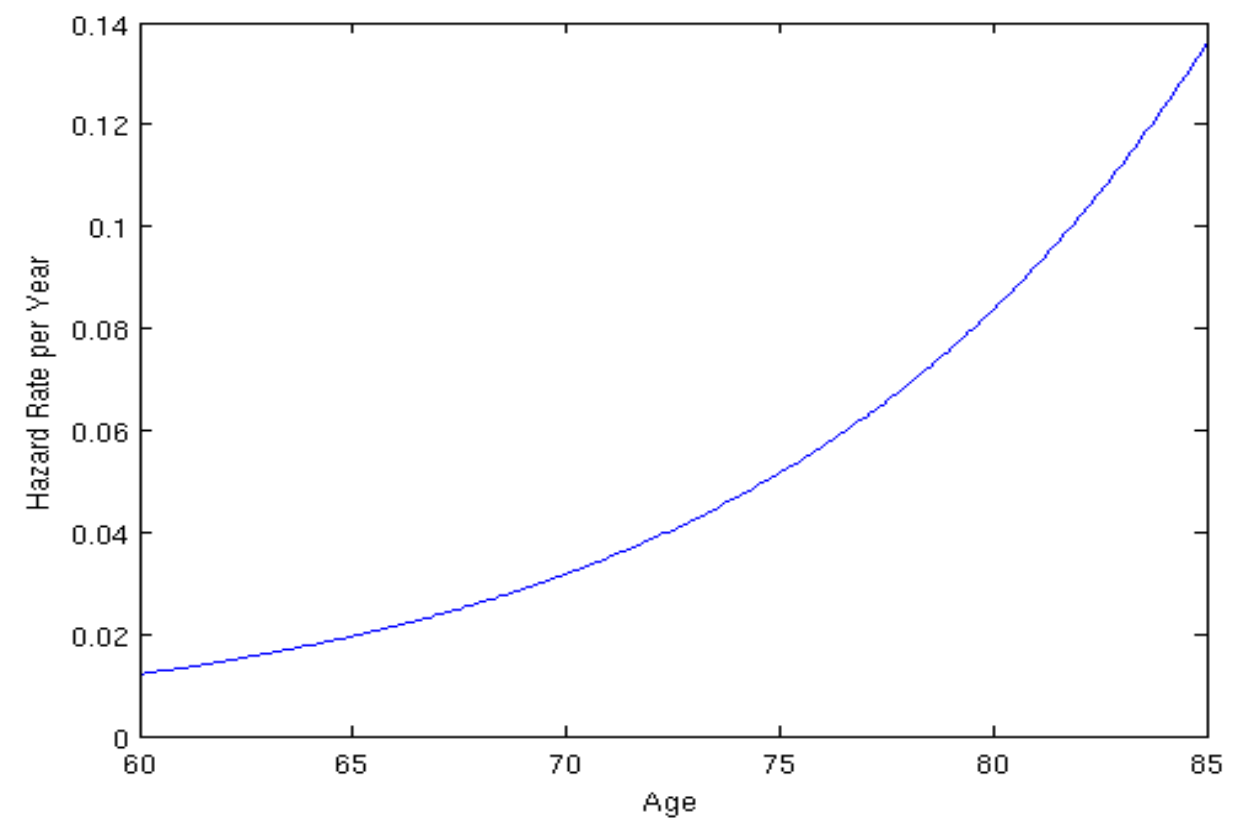

Figure 1: The Gompertz-Makeham Mortality Age Structure Fitted by Dahl and Møller (2006). This is the age-structure used throughout the paper.

time-decreasing mean-reversion level for $Y$. To maintain positive mortality rates we select $\bar{y}=10, y=0.01$ in (1); these values are largely irrelevant for the small volatility $\sigma_{\lambda}$ of $Y$ one expects in practice. For instance in the example below, $\mathbb{P}\left[0.7 \leq Y_{t} \leq 1.3 \forall 0 \leq t \leq T\right]>99.9 \%$.

Interest rates are modeled using the Vasicek (Björk 2004) model under both the physical and market measures,

$$
\left\{\begin{array}{l}
d r_{t}=\kappa_{r}\left(\theta^{\mathbb{P}}-r_{t}\right) d t+\sigma_{r} d W_{t}^{r}, \\
d r_{t}=\kappa_{r}\left(\theta^{\mathbb{Q}}-r_{t}\right) d t+\sigma_{r} d \widetilde{W}_{t}^{r}
\end{array}\right.
$$

Table 1 summarizes all our parameter values. For the insurer's portfolio data we assume that each life insurance policy pays out death benefits of $\$ 100,000$ (thus $I=\$ 10^{11}$ ) and the insurance premiums are set at $\$ 6,000\left(\bar{p}_{I}=6 \cdot 10^{9}\right)$ per annum. Annuities pay at $\$ 40,000$ per year $\left(A=4 \cdot 10^{10}\right)$. Finally, $\beta=5 \%$ of the pool have purchased life insurance. Summarizing, we have (in billions of dollars)

$$
f d t=\left(\alpha \cdot 40 \cdot S_{t}^{A}+5 \cdot \lambda_{t}^{I} S_{t}^{I}-0.3 \cdot S_{t}^{I}\right) d t .
$$

As shown by Gerber (1976), the probability of ruin in a static insurance problem using an exponential premium rule is $\mathrm{e}^{-\gamma x}$, where $\gamma$ is the risk-aversion coefficient and $x$ are the insurer's (initial) reserves. In a dynamic setting one expects ruin to be more likely, but roughly speaking we expect $\mathrm{e}^{-\gamma x} \sim 10^{-5}$ or $\gamma x \sim 5-15$. In our example where the nominal values are in billions of dollars, the reserves are likely to be $x \in\left[10^{8}, 10^{9}\right]$, so we consider $\gamma \in\left[10^{-8}, 10^{-7}\right]$.

In terms of the risk faced by the insurer, we find that under the bond-pricing measure $\mathbb{Q}$, the total annuity payouts have a mean of $\$ 38.47 \mathrm{bn}$ and a standard deviation of $\$ 1.45 \mathrm{bn}$. Most of 


\begin{tabular}{|c|c|}
\hline Parameter & Value \\
\hline$x^{0}$ & 65 \\
$T_{1}$ & 30 \\
$T$ & 20 \\
$a_{\lambda}$ & $1.30 \cdot 10^{-4}$ \\
$b_{\lambda}$ & $3.53 \cdot 10^{-5}$ \\
$c_{\lambda}$ & 1.102 \\
$\sigma_{\lambda}$ & 0.03 \\
$\kappa_{\lambda}$ & 0.2 \\
$\sigma_{r}$ & 0.01 \\
$\kappa_{r}$ & 0.2 \\
$\theta_{r}^{\mathbb{P}}$ & 0.04 \\
$\theta_{r}^{\mathbb{Q}}$ & 0.055 \\
\hline
\end{tabular}

Table 1: Numerical parameter values for main example.

the risk comes from the stochastic interest rates. This is because the parameter $\sigma_{\lambda}=0.03$ is the volatility of $Y$, so that the (time-dependent) volatility of force of mortality $\Lambda\left(t, Y_{t}\right)=\xi\left(x_{0}+t\right) Y_{t}$ is about 10-20 times less and much smaller than $\sigma_{r}=0.01$.

\subsection{Relative Indifference Price of Annuities}

We return to the problem of studying $H^{\alpha A \mid \beta I}$, the relative indifference price of selling annuities to fraction $\alpha$ of the population, given an existing portfolio of $\beta$ life-insurances. Due to the nonlinear structure of (A.59), $H^{\alpha A \mid \beta I}$ does not satisfy an autonomous pde; accordingly to study it one must work directly from the definition.

We fix $\alpha=0.1$ and study the relative price charged for selling $\alpha A$-worth of annuities given a portfolio of $\beta I$-worth of life insurances. We compare the exponential premium rule $H$ to the Sharpe ratio premium $\mathcal{H}$, and also compare the relative prices (with respect to $\beta I$-worth of life insurance contracts) versus the absolute price of selling $\alpha A$ annuities. The results are summarized in Table 2. To see the effect of risk-aversion of the insurer, we also have two benchmarks given by the lower bound in (26) (corresponding to pricing under the minimal martingale measure $\mathbb{Q}$ using "forward" discounting), and the price under no mortality risk:

$$
\bar{H}=\int_{t}^{T} F(r, t ; u) \beta\left(I \Lambda(u, 1)-\bar{p}_{I}\right) \mathrm{e}^{-\int_{t}^{u} \Lambda(s, 1) d s} \cdot S d u .
$$

Note that both of these are linear pricing mechanisms and hence do not incorporate portfolio effects. Table 2 also contains two other pricing mechanisms that will be identified in the next section. These are pricing under the minimal martingale measure $\mathbb{Q}$ using standard discounting, cf. (40), and pricing under the relative martingale measure $\hat{\mathbb{Q}}$, cf. $H^{(0,1)}$ in Theorem 6.1.

Recall that in this example the nominal value of the life-insurance contracts sold is $\$ 5$ billion, and the nominal value of annuities is $\$ 4$ billion per year for twenty years. We see that the insurer should ask for about $\$ 38.5$-41 billion for these annuities, which can be quoted as an annuity factor of about $9.5-10.5$ cents on the dollar paid. We emphasize that such a quote is misleading for $H$ which does not scale linearly in quantity $\alpha$. Since $H^{\alpha A \mid \beta I}$ is convex in $\alpha$, the 


\begin{tabular}{|c|c|c|}
\hline Premium Principle & Price with Low Risk-Aversion & Price with High Risk-Aversion \\
\hline Deterministic $\Lambda$ & 38.47 & --- \\
Minimal $\mathbb{Q}$ via $(40)$ & 38.62 & --- \\
Minimal $\mathbb{Q}$ via $(26)$ & 38.85 & --- \\
Exponential $H^{\alpha A \mid \beta I}$ & 39.20 & 41.44 \\
Exponential $H^{\alpha A}$ & 39.61 & 39.71 \\
Sharpe Ratio $\mathcal{H}^{\alpha A \mid \beta I}$ & 38.75 & 39.59 \\
Sharpe Ratio $\mathcal{H}^{\alpha A}$ & 38.86 & 37.39 \\
Relative $\mathbb{Q}$ & 38.28 & \\
\hline
\end{tabular}

Table 2: Comparison of pricing mechanisms for term annuities. For low risk-aversion we take $\gamma=10^{-8}$ and $\gamma^{S R}=0.25$; for high risk-aversion $\gamma=4 \cdot 10^{-8}$ and $\gamma^{S R}=1$. In all cases, the amount of contracts sold is $\alpha A=\$ 4$ billion per year, and $\beta I=\$ 5$ billion per year. The initial parameters are $r_{0}=0.04, Y_{0}=1, S_{0}=1$.

correct meaning of $H^{\alpha A \mid \beta I}$ is the premium to be charged for selling annuities up to the target proportion $\alpha$ of the pool. If sales are less, say $\alpha^{\prime}$, then the insurer will have a surplus since the value of the annuities sold will be less: $H^{\alpha^{\prime} A \mid \beta I} \leq \frac{\alpha^{\prime}}{\alpha} H^{\alpha A \mid \beta I}$. If the insurer wants to sell more than $\alpha A$ annuities, then a new (relative) price will need to be computed.

As Table 2 shows, the cross-hedging effect in $H$ is non-trivial: it reduces reserves by over $\$ 0.4 b n$ for a weakly risk-averse insurer and by over $\$ 2 b n$ for a highly risk-averse one. While these numbers are small compared to the total reserves needed, they are very significant in terms of the extra premium charged due to systematic stochastic mortality. Compared to no mortality risk (the "deterministic" premium of $\bar{H}=\$ 38.47 \mathrm{bn}$ ), the mortality-risk premium is $39.61-38.47$ with $\gamma=10^{-8}$ and $43.71-38.47$ with $\gamma=4 \cdot 10^{-8}$. Thus, the portfolio cross-hedge cuts this premium by $36 \%$ and $43 \%$ respectively. A similar cross-hedging effect on the order of $30 \%$ is present for the Sharpe ratio pricing. Let us recall however that the latter is not always able to capture the portfolio effect.

\subsection{Relative Hedging Demand}

The relative hedging policy of selling $\alpha$ annuities is given by $\pi^{\alpha A+\beta I \mid \beta I}=\pi^{\alpha A+\beta I}-\pi^{\beta I}$ where $\pi^{\cdot}$ is the optimal control in the utility maximization problem of $U^{f}$. Recall that $\pi_{t}^{\alpha A+\beta I \mid \beta I}$ represents the dollar amount that the investor should invest in bonds at time $t$, given the values of other state variables. Economically, $\pi^{\alpha A+\beta I \mid \beta I}$ represents the effect that the sale of $\alpha$ annuities has on the optimal investment strategy of the insurer. Applying (23) and cancelling out terms independent of $f$ we compute

$$
\begin{aligned}
\pi_{t}^{\alpha A+\beta I \mid \beta I}=\frac{1}{C\left(t, T_{1}\right)}\left[C ( t , T ) \left(X_{t}^{\alpha A+\beta I}-X_{t}^{\beta I}\right.\right. & \left.+H^{\beta I}\left(Y_{t}, r_{t}, S_{t}, t\right)-H^{\alpha A+\beta I}\left(Y_{t}, r_{t}, S_{t}, t\right)\right) \\
& \left.+H_{r}^{\beta I}\left(Y_{t}, r_{t}, S_{t}, t\right)-H_{r}^{\alpha A+\beta I}\left(Y_{t}, r_{t}, S_{t}, t\right)\right] .
\end{aligned}
$$

Hence, the relative hedge of annuities is given by: (i) a demand due to the different net surplus from selling annuities, (ii) net interest-rate Delta caused by adding the annuities to the overall insurance portfolio. If we suppose that a firm starts with an initial endowment $x_{0}$ at time 
0 and then sells mortality contingent claims according to the exponential premium principle $H$, then $X_{0}^{\alpha A+\beta I}-H^{\alpha A+\beta I}\left(Y_{0}, r_{0}, S_{0}, 0\right)=X_{0}^{\beta I}-H^{\beta I}\left(Y_{0}, r_{0}, S_{0}, 0\right)$. Thus, we would have $\pi_{0}^{\alpha A+\beta I \mid \beta I}=\left(H_{r}^{\beta I}-H_{r}^{\alpha A+\beta I}\right)\left(Y_{0}, r_{0}, S_{0}, 0\right)$, which is illustrated in the left panel of Figure 2 for the parameters of Table 1 . We see that the firm needs to invest about $60 \%-70 \%$ of its reserves in the $T_{1}$-bond. The amount increases for smaller $Y$ (which increase the expected liability as a result of reduced mortality) or smaller $r$ (which reduces the discounting of future payments).

The right panel of Figure 2 shows the excess demand resulting from stochastic mortality. As we can see, this demand is on the order of $10-15 \%$ of the total invested in bonds; intuitively stochastic $Y$ increases the price of contingent claims, which is then more sensitive to interest rates and consequently results in higher hedging demand.
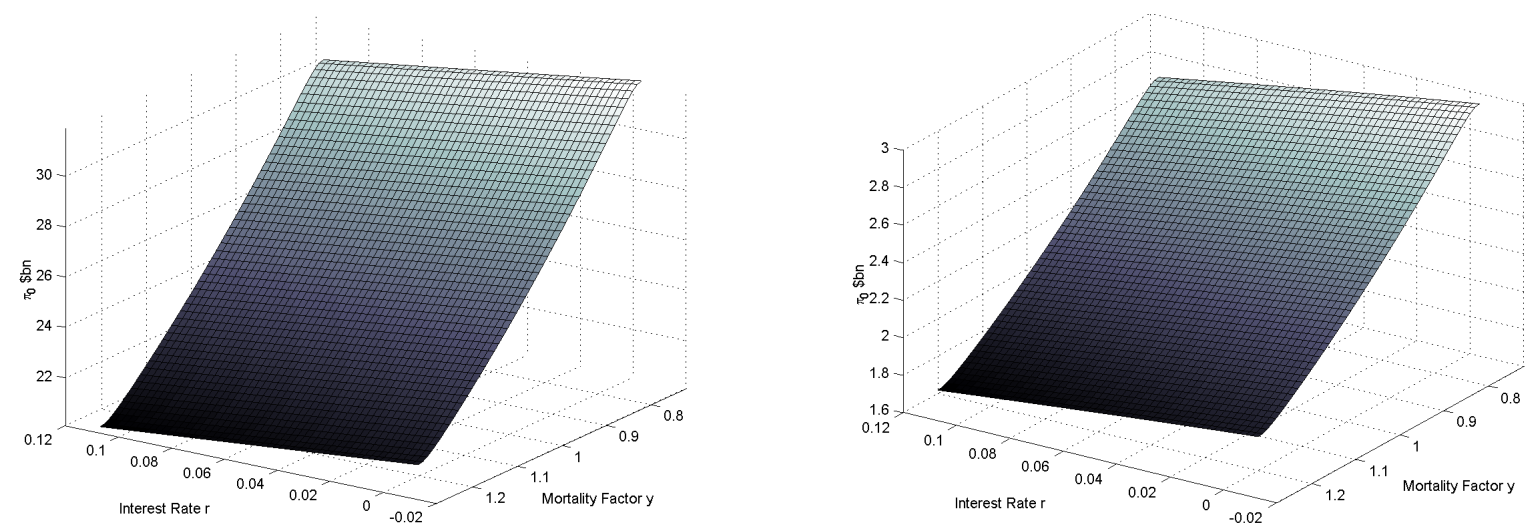

Figure 2: Left panel shows initial Hedging Demand $\pi_{0}^{\alpha A+\beta I \mid \beta I}$. Right panel shows the difference $\pi_{0}-\pi_{0}^{\sigma_{\lambda}=0}$ in hedging demand compared to having deterministic mortality.

Remark 5.1 One could also consider the converse problem: an insurer has sold temporary annuities to a proportion $\alpha$ of the pool and would like to add $\beta$ life-insurances. Since lifeinsurance has a continuously paid premium, we are now interested in finding such an indifference premium $\tilde{p}^{I}$. The latter should satisfy

$$
U^{\alpha A}(x, y, r, S, t)=U^{\alpha A+\beta I}\left(x, y, r, S, t ; \tilde{p}^{I}\right)
$$

where we explicitly indicate the dependence of $U^{\alpha A+\beta I}$ from Theorem 3.2 on the continuous insurance premium. Proceeding like in previous subsection we then find that $\tilde{p}^{I}$ solves

$$
H^{\alpha A \mid \beta I}\left(y, r, S, t ; \tilde{p}^{I}\right)-H^{\alpha A}(y, r, S, t)=0 .
$$

No further simplification is possible and to find $\tilde{p}^{I}$ one must numerically solve the two semilinear pdes and search for the zero of the corresponding function. For instance, for the numerical values above and $\gamma=4 \cdot 10^{-8}$ we find $\tilde{p}^{I}=5.3 \%$. Much of the ensuing analysis can be carried out, but due to the above difficulty we focus on the original setting.

\section{Asymptotic Analysis}

In this section we study the asymptotic correction terms that highlight the various nonlinearities present in the exponential premium framework. After studying the dependence of $H^{\alpha A \mid \beta I}$ on 
quantity of annuities sold $\alpha$, we analyze the effect of stochastic mortality in Section 6.2 and the effect of insurer's risk-aversion in Section 6.3. In each case we use the asymptotic expansions to obtain the optimal proportion $\alpha^{*}$ of annuities to be sold. These expressions are justified because all the quantities are expected to be small (but not too small that individual mortality events start to matter) with respect to the size of the global pool $S$. Proofs are again delegated to the Appendix.

\subsection{Expansion in Quantity $\alpha$}

Our first step is to understand how $H^{\alpha A \mid \beta I}$ depends on $\alpha$. Since the pricing rule is nonproportional, $H^{\alpha A \mid \beta I}$ is not linear in $\alpha$, see Corollary 3.2 .

Theorem 6.1 Let $\hat{\mathbb{Q}}$ be the $\mathbb{P}$-equivalent probability measure with the Radon-Nikodym derivative

$$
\left.\frac{d \hat{\mathbb{Q}}}{d \mathbb{P}}\right|_{\mathcal{F}_{T}}=\exp \left(\int_{0}^{T} \frac{\sigma^{2} \gamma}{F(r, s ; T)} H_{y}^{\beta I}\left(Y_{s}, r_{s}, S_{s}, s\right) d W_{s}-\frac{1}{2} \int_{0}^{T} \frac{\sigma^{4} \gamma^{2}}{F(r, s ; T)^{2}} H_{y}^{\beta I}\left(Y_{s}, r_{s}, S_{s}, s\right)^{2} d s\right) .
$$

Define

$$
\left\{\begin{array}{l}
H^{(1)}(y, r, S, t)=\mathbb{E}^{\hat{\mathbb{Q}}}\left[\int_{t}^{T} A S_{s} \mathrm{e}^{-\int_{t}^{s} r_{u} d u} d s \mid r_{t}=r, Y_{t}=y, S_{t}=S\right] \\
H^{(2)}(y, r, S, t)=\mathbb{E}^{\hat{\mathbb{Q}}}\left[\int_{t}^{T} \frac{1}{2} \sigma^{2} \frac{\gamma}{F\left(r_{s}, s, T_{1}\right)}\left(H_{y}^{(1)}(\cdot, s)\right)^{2} \mathrm{e}^{-\int_{t}^{s} r_{u} d u} d s \mid r_{t}=r, Y_{t}=y, S_{t}=S\right] \geq 0 .
\end{array}\right.
$$

Then $H^{\alpha A \mid \beta I}=\alpha H^{(1)}+\alpha^{2} H^{(2)}+\mathcal{O}\left(\alpha^{3}\right)$ and

$$
\alpha^{*} \simeq \frac{A p-H^{(1)}(y, r, S, t)}{2 H^{(2)}(y, r, S, t)} .
$$

In particular, $\alpha^{*}$ is linear in the market price $p$ as expected. We call the measure $\hat{\mathbb{Q}}$ the relative hedging measure, since it depends on the indifference price of the $\beta$ life-insurance contracts the company already holds (more precisely on the mortality-sensitivity of the latter). Thus, $H^{(1)}$ is the expected size of annuity payout under the relative indifference measure $\hat{\mathbb{Q}}$. The term $H^{(2)}$ can be viewed as a convexity correction to the "risk-adjusted" price $H^{(1)}$, which we see is in turn proportional to the $Y$-sensitivity of the former. Moreover, we see that $H^{(2)}$ is linearly proportional to the risk-aversion parameter $\gamma$.

Since value of life insurance increases when $Y$ increases, $H_{y}^{\beta I}>0$ and therefore under $\hat{\mathbb{Q}}$ the drift of $Y$ is larger; recall that $d Y_{t}=\left(\mu+\frac{\sigma^{2} \gamma}{F\left(r_{t}, t ; T\right.} H_{y}^{\beta I}\left(Y_{t}, r_{t}, S_{t}, t\right)\right) d t+\sigma d W_{t}^{\hat{\mathbb{Q}}}$. Consequently, under $\hat{\mathbb{Q}}$ the value of annuities is less, indicating the cross-hedge between life insurance and annuities. In our numerical example for $\gamma=4 \cdot 10^{-8}$ we compute

$$
\left\{\begin{array}{l}
H^{(1)}\left(y_{0}, r_{0}, 1,0\right)=373.86 \\
H^{(2)}\left(y_{0}, r_{0}, 1,0\right)=281.36
\end{array}\right\} \quad \Longrightarrow \quad \alpha^{*} \simeq 0.071 p-0.6644
$$


where $p$ is the annuity factor (price charged for delivering $\$ 1$ to annuitant). For instance, if the market price is set using the risk-neutral rule $H^{\mathbb{Q}}=386.23 \Leftrightarrow p=H^{\mathbb{Q}} / A=9.655$ then $\alpha^{*}=2.11 \%$, so the insurer should sell $\alpha^{*} A=\$ 0.844$ billion worth of annuities. For comparison, when $\gamma=10^{-8}, \alpha^{*}=2.05 \%$, so the result is not very sensitive to $\gamma$. Using the measure $\hat{\mathbb{Q}}$ to calculate premiums on contracts could be an attractive possibility - the resulting premium rule is linear in quantity, but still captures relative hedging and incorporates the risk-aversion through $\gamma$ appearing in (31). The semi-linear pde that defines $H_{y}^{\beta I}$ can be solved once offline and then used for linear pricing via (32).

Figure 3 shows the resulting quadratic approximation over a range of $\alpha$ 's compared to exact values of $H^{\alpha A+\beta I}$. We observe that the quadratic term is quite large so that the cubic term is also needed for $\alpha>0.1$.

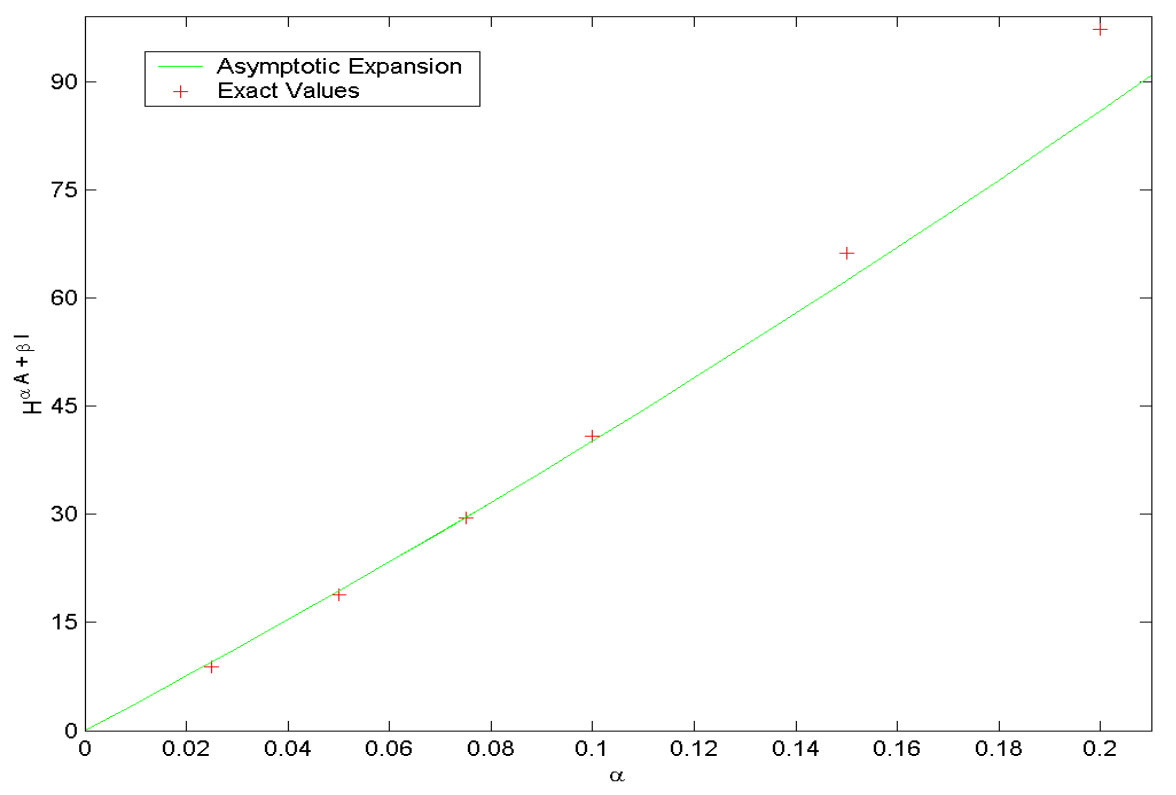

Figure 3: $\alpha$-Expansion Results. The quadratic approximation of Theorem 6.1 is shown in green, and exact values from solving (20) directly are the red crosses. We use the parameter values of Table 1 and $\gamma=4 \cdot 10^{-8}$.

\subsection{Slow Mortality Rate Approximation}

It is clear that mortality rates change very slowly over time. This is the reason that many practitioners continue to assume deterministic mortality tables and simply update the tables every few years for their hedging purposes. Thus, in our stochastic mortality model, it is important to understand the effect of stochasticity of mortality factor $Y$. This can be conveniently carried out in our pde framework. In particular, we are able to carry out asymptotic analysis that shows the first- and second-order effects of the stochastic assumption.

To do so, in this section we will assume that the process $Y_{t}$ driving the intensity is a slowly varying diffusion, i.e.,

$$
d Y_{t}=\delta \mu\left(Y_{t}\right) d t+\sqrt{\delta} \sigma d W_{t}, \quad Y_{0}=y
$$


in which $\delta>0$ is a timescaling parameter. The terms of $\delta$ in (34) are selected so that the meanreversion timescale of $Y$ remains the same and only the magnitude of fluctuations is affected. The scale $\delta$ should be chosen so that the fluctuations of $r$ and $\Lambda$ are of comparable frequency; for mortality rates and interest rates this means that $\delta \sim 0.1$.

\section{Theorem 6.2 Let}

$$
\begin{aligned}
& \widetilde{H}^{(0,0)}(y, r, S, t)=\int_{t}^{T} F(r, t ; u) \beta\left(I \Lambda(u, y)-\bar{p}_{I}\right) \mathrm{e}^{-\int_{t}^{u} \Lambda(s, y) d s} \cdot S d u, \\
& \widetilde{H}^{(0,1)}(y, r, S, t)=\int_{t}^{T} F(r, t ; u) A \mathrm{e}^{-\int_{t}^{u} \Lambda(s, y) d s} \cdot S d u
\end{aligned}
$$

and

$$
\left\{\begin{array}{l}
\widetilde{H}^{(1,1)}=\mathbb{E}_{t}^{\mathbb{Q}}\left[\int_{t}^{T} \mathrm{e}^{-\int_{t}^{s} r_{u} d u}\left\{\mu \widetilde{H}_{y}^{(0,1)}+\frac{1}{2} \sigma^{2}\left(\widetilde{H}_{y y}^{(0,1)}+\frac{2 \gamma \widetilde{H}_{y}^{(0,0)} \widetilde{H}_{y}^{(0,1)}}{F\left(r_{u}, u ; T\right)}\right)\right\} d u \mid r_{t}=r\right], \\
\widetilde{H}^{(1,2)}=\mathbb{E}_{t}^{\mathbb{Q}}\left[\int_{t}^{T} \mathrm{e}^{-\int_{t}^{s} r_{u} d u} \frac{\gamma \sigma^{2}}{2 F(r, u ; T)}\left(\widetilde{H}_{y}^{(0,1)}\right)^{2} d u \mid r_{t}=r\right] .
\end{array}\right.
$$

Then $H^{\alpha A \mid \beta I}(y, r, S, t)=\alpha \widetilde{H}^{(0,1)}+\delta\left(\alpha \widetilde{H}^{(1,1)}+\alpha^{2} \widetilde{H}^{(1,2)}\right)+\mathcal{O}\left(\delta^{2}\right)$ and for $\delta$ small,

$$
\alpha^{\delta} \approx \frac{A p-\widetilde{H}^{(0,1)}-\delta \widetilde{H}^{(1,1)}}{2 \delta \widetilde{H}^{(1,2)}} .
$$

In (36), the arguments of all the functions inside the expectation is $\left(y, r_{t}, \mathrm{e}^{-\int_{0}^{t} \Lambda(s, y) d s} \cdot S, t\right)$, so the only random variable is $r$. Note that $\widetilde{H}^{(0,1)}\left(\right.$ resp. $\left.\widetilde{H}^{(0,0)}\right)$ is the price of $\alpha$ annuities $(\beta$ life-insurances) when the population's hazard rate is deterministic $\Lambda(u, y)$. In that case, the insurer has no risks left, since she can perfectly hedge away interest rate risk by trading in the $T_{1}$-bond. Thus, the price of the contract is simply the present value of future cashflows, which is obtained by discounting the deterministic mortality payments by today's bond prices. The next three functions are correction terms, which use the bond risk-neutral measure $\mathbb{Q}$. We see that their value is proportional to mortality-sensitivity of the former expressions.

In the expression for $H^{\alpha A \mid \beta I}$, we see that the $\mathcal{O}(\delta)$ term consists of three parts: $(1)$ the $\widetilde{H}_{y}^{(0,1)}$ and $\widetilde{H}_{y y}^{(0,1)}$ terms related to the $Y$-sensitivity of the deterministic payoff; $(2)$ the $\left(\widetilde{H}_{y}^{(0,1)}\right)^{2}$ term due to nonlinear utility preferences; $(3)$ the $\widetilde{H}_{y}^{(0,0)} \widetilde{H}_{y}^{(0,1)}$ term due to relative hedging with $\beta I$ life insurances.

In the numerical example considered, we take $\delta=0.1$ and work with

$$
d Y_{t}=2 \delta\left(1-Y_{t}\right) d t+0.095 \sqrt{\delta} d W_{t}
$$

We then obtain for $r_{0}=0.04, Y_{0}=1, S_{0}=1, \gamma=4 \cdot 10^{-8}$

$$
\left\{\begin{array}{ll}
\widetilde{H}^{(0,0)}=-0.790, & \widetilde{H}^{(0,1)}=384.67 \\
\widetilde{H}^{(1,1)}=-953 & \widetilde{H}^{(1,2)}=20200 .
\end{array}\right\} \quad \Longrightarrow \quad \alpha^{\delta} \simeq 0.0099 p-0.0716 .
$$

If the market price is set to the deterministic price $\bar{H}=38.47 \Leftrightarrow p=9.6175$ then $\alpha^{\delta}=2.35 \%$. Let us remark that in $(36), \widetilde{H}_{y}^{(0,0)} \geq 0$ and all the second derivatives are positive (since insurance 
value increases when mortality increases), while $\widetilde{H}_{y}^{0,1}$ is strongly negative (annuity value drops with higher mortality). The latter dominates the RHS of the second line of (36) and causes $\widetilde{H}^{(1,1)}$ to be negative. We also observe that $\widetilde{H}^{(1,1)} / \widetilde{H}^{(1,2)}$ is almost constant with respect to $\gamma$ so that the answer in (37) does not depend much on the risk-aversion selected.

\subsection{Expansion in the Risk Aversion $\gamma$}

In order to understand the effect of the risk aversion parameter $\gamma$ on the exponential premia , we will write out the asymptotic dependence of $H$ on $\gamma$.

Theorem 6.3 Let

$$
\left\{\begin{array}{l}
\widehat{H}^{(0,0)}(y, r, S, t)=\mathbb{E}_{t}^{\mathbb{Q}}\left[\int_{t}^{T} F(r, t ; u)\left(\beta I \Lambda\left(u, Y_{u}\right) S_{u}-\beta \bar{p}_{I} S_{u}\right) d u\right] \\
\widehat{H}^{(0,1)}(y, r, S, t)=\mathbb{E}_{t}^{\mathbb{Q}}\left[\int_{t}^{T} F(r, t ; u) A S_{u} d u\right] \\
\widehat{H}^{(1,1)}(y, r, S, t)=\mathbb{E}_{t}^{\mathbb{Q}}\left[\int_{t}^{T} \mathrm{e}^{-\int_{t}^{s} r_{u} d u} \frac{\sigma^{2}}{F\left(r_{u}, u ; T\right)} \widehat{H}_{y}^{(0,0)} \widehat{H}_{y}^{(0,1)} d u\right] \\
\widehat{H}^{(1,2)}(y, r, S, t)=\mathbb{E}_{t}^{\mathbb{Q}}\left[\int_{t}^{T} \mathrm{e}^{-\int_{t}^{s} r_{u} d u} \frac{\sigma^{2}}{2 F\left(r_{u}, u ; T\right)}\left(\widehat{H}_{y}^{(0,1)}\right)^{2} d u\right]
\end{array}\right.
$$

and

$$
\begin{aligned}
\widehat{H}^{(2)}(y, r, S, t)=\mathbb{E}_{t}^{\mathbb{Q}}\left[\int _ { t } ^ { T } \mathrm { e } ^ { - \int _ { t } ^ { s } r _ { u } d u } \frac { 3 \sigma ^ { 4 } } { 2 F ^ { 2 } ( r _ { u } , u ; T ) } \left\{2 \alpha \widehat{H}_{y}^{(0,0)} \widehat{H}_{y y}^{(0,0)} \widehat{H}_{y}^{(0,1)}+\alpha\left(\widehat{H}_{y}^{(0,0)}\right)^{2} \widehat{H}_{y y}^{(0,1)}\right.\right. \\
\left.\left.+\alpha^{2} \widehat{H}_{y y}^{(0,0)}\left(\widehat{H}_{y}^{(0,1)}\right)^{2}+2 \alpha^{2} \widehat{H}_{y}^{(0,0)} \widehat{H}_{y}^{(0,1)} \widehat{H}_{y y}^{(0,1)}+\alpha^{3}\left(\widehat{H}_{y}^{(0,1)}\right)^{2} \widehat{H}_{y y}^{(0,1)}\right\}\right] .
\end{aligned}
$$

Then,

$$
H^{\alpha A \mid \beta I}=\alpha \widehat{H}^{(0,1)}+\gamma\left(\alpha \widehat{H}^{(1,1)}+\alpha^{2} \widehat{H}^{(1,2)}\right)+\gamma^{2} \widehat{H}^{(2)}+\mathcal{O}\left(\gamma^{3}\right)
$$

and

$$
\alpha^{\gamma} \approx \frac{A p-\widehat{H}^{(0,1)}-\gamma \widehat{H}^{(1,1)}}{2 \gamma \widehat{H}^{(1,2)}} .
$$

The first two terms represent the risk-neutral (i.e. when no risk premium is given to stochastic mortality) price of life insurance and annuities. The third term $\widehat{H}^{(1,1)}$ will be negative for our case study and corrects for the portfolio cross-hedge. The fourth term $\widehat{H}^{(1,2)}$ adjusts for the nonlinear risk-preferences of the insurer by counting the variance of the sensitivity of reserves to mortality.

In our numerical example we find that

$$
\left\{\begin{array}{ll}
\widehat{H}^{(0,0)}=-0.799 & \widehat{H}^{(0,1)}=386.21 \\
\widehat{H}^{(1,1)}=-7.12 \cdot 10^{-4} & \widehat{H}^{(1,2)}=1.77 \cdot 10^{-2}
\end{array}\right\} \quad \Longrightarrow \quad \alpha^{\gamma} \simeq 28.249 p-272.727
$$

for $\gamma=4 \cdot 10^{-8}$. For instance if $p=9.655$ is equal to the risk-neutral price $H^{\mathbb{Q}}$ then $\alpha^{\gamma}=2.01 \%$. 


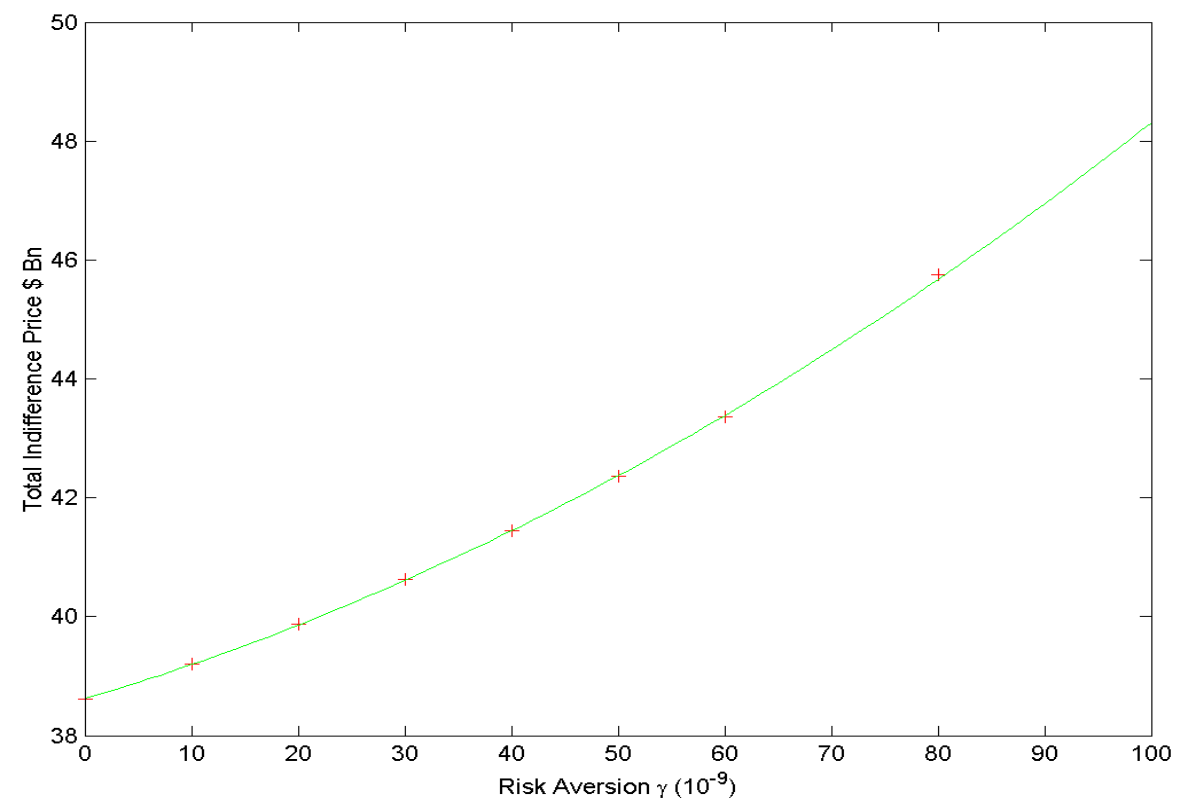

Figure 4: $\gamma$-Expansion Results from Theorem 6.3. The quadratic approximation is indicated by the (green) parabola; the (red) crosses represent actual values of $H^{\alpha A \mid \beta I}$ computed using an explicit finite-difference method for (20).

Returning to $H^{\alpha A \mid \beta I}$ in the numerical setting of Section 5 , we find that for $\alpha=0.1, H^{\alpha A \mid \beta I}(\cdot ; \gamma) \simeq$ $38.62+0.0531 \gamma+0.000438 \gamma^{2}$. Figure 4 shows that the accuracy of the above expansion is excellent in the range of interest.

Since we know that $H$ increases in $\gamma$, the linear zero-order term $\alpha \widehat{H}^{(0,1)}$ is a lower bound on $H$. We summarize this observation in the following

Corollary 6.1 For any mortality-contingent claim $f$ we have the alternative lower bound (cf. (26).)

$$
H(y, r, S, t) \geq \mathbb{E}_{t}^{\mathbb{Q}}\left[\int_{t}^{T} F(r, t ; u) f\left(\Lambda\left(u, Y_{u}\right), \vec{S}_{u}\right) d u\right]
$$

Note that the right-hand-side of (40) is the standard discounted expected benefit under the minimal martingale measure $\mathbb{Q}$. In fact, in our numerical example (40) provides a worse lower bound than the LHS of (26), cf. Table 2 .

\subsection{Monopoly Insurer}

It is also possible to assume that the insurer is a monopoly in the annuity market. Thus, the insurer is free to choose the annuity premium $p_{A}$. However, the demand for annuities is limited and declines as $p_{A}$ increases. Namely, a fraction $D\left(p_{A}\right)$ of the pool are willing to pay an upfront premium $p_{A}$ for the annuity (we disregard issues of self-selection and assume that the quality of these contracts is independent of $p_{A}$ ). Again, the firm would like to maximize value from selling 
$D\left(p_{A}\right)$ annuity contracts at premium $p_{A}$, given its existing portfolio. In this setting, the firm maximizes its profit via

$$
p_{A}^{*}=\operatorname{argmax}_{p}\left(p \cdot D(p)-H^{D(p) A \mid \beta I}\right) .
$$

In order to solve (41) we postulate that the demand is linear and satisfies $D(p)=\alpha_{D}-\beta_{D} p$ in the price-range of interest. It is then possible to find approximations to the maximizer $p^{*}$ using the asymptotic expansions of this section.

First, in the context of Section 6.2, for small values of $\delta>0$ and using (36), the problem can be reformulated as

$$
\arg \max _{p}\left(\alpha_{D}-\beta_{D} p\right) p-\left(\left(\alpha_{D}-\beta_{D} p\right) \widetilde{H}^{(0,1)}+\delta\left[\left(\alpha_{D}-\beta_{D} p\right) \widetilde{H}^{(1,1)}+\left(\left(\alpha_{D}-\beta_{D} p\right)\right)^{2} \widetilde{H}^{(1,2)}\right]\right)+\mathcal{O}\left(\delta^{2}\right) .
$$

The optimizer of this expression is easily found to be

$$
p^{\delta} \approx \frac{\alpha_{D}+\beta_{D} \widetilde{H}^{(0,1)}+\delta\left(\beta_{D} \widetilde{H}^{(1,1)}+2 \alpha_{D} \beta_{D} \widetilde{H}^{(1,2)}\right)}{2 \beta_{D}\left(1+2 \delta \beta_{D} \widetilde{H}^{(1,2)}\right)} .
$$

Similarly, in the context of Section 6.3 , for small values of risk-aversion $\gamma$, we find

$$
p^{\gamma} \approx \frac{\alpha_{D}+\beta_{D} \widehat{H}^{(0,1)}+\gamma\left(\beta_{D} \widetilde{H}^{(1,1)}+2 \alpha_{D} \beta_{D} \widehat{H}^{(1,2)}\right)}{2 \beta_{D}\left(1+2 \gamma \beta_{D} \widehat{H}^{(1,2)}\right)}
$$

with $\widehat{H}$ 's as given in (38).

\section{$7 \quad$ Effect of Mortality Age Structure}

In the more realistic setting, the mortality improvement process of the annuity pool is different from mortality improvement of life insurance holders. In particular, the uncertainty of the insured young lives is smaller than the risk of the older annuitants. Moreover, the two groups maybe affected differently by various mortality developments (e.g. cure for AIDS, new cancer drugs, etc.). We model this situation by introducing a bivariate mortality process $\left(Y^{A}, Y^{I}\right)$ evolving according to

$$
d Y_{t}^{a}=\mu^{a}\left(Y_{t}^{a}\right) d t+\sigma^{a} d W_{t}^{a}, \quad Y_{0}^{a}=y_{a}, \quad a \in\{A, I\}
$$

with $d\left[W^{A}, W^{I}\right]_{t}=\rho d t$ and $\rho \in[-1,1]$ the correlation parameter. To focus again on the stochastic aspect, we will mimic the cohort-based approach and postulate that the shrinkage rate of the survivor fractions $\vec{S}_{t}=\left(S_{t}^{A}, S_{t}^{I}\right)$ is given directly by $\Lambda^{a}\left(t, Y_{t}^{a}\right), a \in\{A, I\}$ with

$$
S_{t}^{a}=\mathrm{e}^{-\int_{0}^{t} \Lambda^{a}\left(s, Y_{s}^{a}\right) d s}, \quad a \in\{A, I\} .
$$

The age-distribution of the pools is thus absorbed into the time-dependence of $\Lambda^{a}$ 's. The presence of two sources of risk reduces the relative hedge between life insurance and annuities, but at the same time leads to diversification of mortality uncertainty. 
In this setup we now have five stochastic factors, namely $\left(r, Y^{A}, Y^{I}, S^{A}, S^{I}\right)$ which make direct analysis unwieldy. As a result we focus on understanding the effect of the correlation parameter $\rho$; this is also the reason for approaching this model via (45) and (46). Following the same steps as in the previous section one can show that the indifference price of $f$ for the two-pool case satisfies

$$
D_{t}+\mathcal{L}_{r}^{\mathbb{Q}} D+\widetilde{\mathcal{L}}_{Y} D+\frac{1}{2}\left(\sigma^{A}\right)^{2} \frac{\gamma}{F} D_{y_{A}}^{2}+\frac{1}{2}\left(\sigma^{I}\right)^{2} \frac{\gamma}{F} D_{y_{I}}^{2}+\rho \sigma^{A} \sigma^{I} \frac{\gamma}{F} D_{y_{A}} D_{y_{I}}+\widetilde{\mathcal{L}}_{S} D+f-r D=0
$$

with the terminal condition $D\left(y_{A}, y_{I}, r, S^{A}, S^{I}, T\right)=0$, in which

$$
\widetilde{\mathcal{L}}_{Y} \triangleq \mu^{A} \partial_{y_{A}}+\mu^{I} \partial_{y_{I}}+\frac{1}{2}\left(\sigma^{A}\right)^{2} \partial_{y_{A} y_{A}}+\frac{1}{2}\left(\sigma^{I}\right)^{2} \partial_{y_{I} y_{I}}+\rho \sigma^{A} \sigma^{I} \partial_{y_{A} y_{I}}
$$

and

$$
\widetilde{\mathcal{L}}_{S} \triangleq-\Lambda^{A} S^{A} \cdot \partial_{S^{A}}-\Lambda^{I} S^{I} \partial_{S^{I}}
$$

The upper and lower bounds of (26) still apply. Note that the lower bound is linear and therefore is not affected by the correlation parameter $\rho$. We find that for reasonable values of $\gamma$, the upper bound changes by less than $1 \%$ for different values of $\rho$, showing little sensitivity.

\section{Slow Mortality Rate Approximation}

In this section, we quantify the affect of the correlation of the hazard rate of the two pools on the exponential premium $H^{\alpha A \mid \beta I}$ and the approximate solution to (12). Similar to Section 6.2, we assume that processes $Y_{t}^{a}, a \in\{A, I\}$, driving the intensities are slowly varying

$$
d Y_{t}^{a}=\delta \mu^{a}\left(Y_{t}^{a}\right) d t+\sqrt{\delta} \sigma^{a} d W_{t}^{a},
$$

with initial conditions $Y_{0}^{A}=y_{0}^{A}$ and $Y_{0}^{I}=y_{0}^{I}$.

Theorem 7.1 Let

$$
\left\{\begin{aligned}
D^{(0,0)}\left(y_{I}, r, S^{I}, t\right) & =\int_{t}^{T} F(r, t ; u) \beta\left(I \Lambda\left(u, y_{I}\right)-\bar{p}_{I}\right) \mathrm{e}^{-\int_{t}^{u} \Lambda\left(s, y_{I}\right) d s} \cdot S^{I} d u \\
D^{(0,1)}\left(y_{A}, r, S^{A}, t\right) & =\int_{t}^{T} F(r, t ; u) A \mathrm{e}^{-\int_{t}^{u} \Lambda\left(s, y_{A}\right) d s} \cdot S^{A} d u,
\end{aligned}\right.
$$

and

$$
\left\{\begin{array}{l}
D^{(1,1)}=\mathbb{E}_{t}^{\mathbb{Q}}\left[\int_{t}^{T} \mathrm{e}^{-\int_{t}^{s} r_{u} d u}\left\{\mu^{A} D_{y_{A}}^{(0,1)}+\frac{1}{2}\left(\sigma^{A}\right)^{2}\left(D_{y_{A} y_{A}}^{(0,1)}\right)+\rho \sigma^{I} \sigma^{A} \frac{\gamma D_{y_{I}}^{(0,0)} D_{y_{A}}^{(0,1)}}{F\left(r_{u}, u ; T\right)}\right\} d u \mid r_{t}=r\right] \\
D^{(1,2)}=\mathbb{E}_{t}^{\mathbb{Q}}\left[\int_{t}^{T} \mathrm{e}^{-\int_{t}^{s} r_{u} d u} \frac{\gamma\left(\sigma^{A}\right)^{2}}{2 F\left(r_{u}, u ; T\right)}\left(D_{y_{A}}^{(0,1)}\right)^{2} d u \mid r_{t}=r\right] .
\end{array}\right.
$$

Then $H^{\alpha A \mid \beta I}=\alpha D^{(0,1)}+\delta\left(\alpha D^{(1,1)}+\alpha^{2} D^{(1,2)}\right)+\mathcal{O}\left(\delta^{2}\right)$ and for $\delta$ small we have $\alpha^{\delta}$ and $p^{\delta}$ as in (37) and (43) upon replacing every $\widetilde{H}$ term by a corresponding D-term.

Again, the first two terms correspond to price of life insurance and annuities under deterministic $Y^{A}$ and $Y^{I}$ processes and the last two terms are first order stochasticity corrections. The key observation that $\rho$ appears only in $D^{(1,1)}$ and the latter is in fact linear in $\rho$. 


\section{Numerical Example}

To isolate the effect of age-structure correlation parameter $\rho$, we take $\sigma^{I}=\sigma^{A}=\sigma, \mu^{I}=\mu^{A}=\mu$, $y_{0}^{I}=y_{0}^{A}=1$, like in the original example with one pool. In that setting, the one-cohort results directly correspond to $\rho=1$. Moreover, we notice that $D^{(0,0)}=\widetilde{H}^{(0,0)}, D^{(0,1)}=\widetilde{H}^{(0,1)}, D^{(1,0)}=$ $\widetilde{H}^{(1,0)}, D^{(1,2)}=\widetilde{H}^{(1,2)}$ with the only difference being in the $D^{(1,1)}$ term. We observe that $D^{(1,1)}$ is linear in $\rho$, and to emphasize the dependence write $D^{(1,1)}(\rho)$. As stated above, $\widetilde{H}^{(1,1)}=D^{(1,1)}(1)$. Furthermore, $D^{(1,1)}$ is decreasing in $\rho$, because $D_{y}^{(0,0)}>0$ and $D_{y}^{(0,1)}<0$, so the last term in the middle case of (A.92) is negative.

In the numerical example stated we find that $D^{(1,1)}(\rho) \simeq 1.53-474 \rho$, so that the optimal $\alpha$ in (37) for $A p=D^{(0,1)}$ is $\alpha^{*}(\rho)=0.0235 \rho-3.78 \cdot 10^{-5}$. We see that the correlation plays a major role in determining optimal amount of annuities to sell (the higher $\rho$, the more sales are optimal) and that essentially the amount of cross-hedging is proportional to $\rho$. This is a convenient conclusion since one may compute the one-dimensional case $\rho=1$ and then prorate by the postulated pool correlation $\rho$.

\section{Small Values of Risk Aversion}

On the other hand, when we perform an expansion in the risk aversion parameter $\gamma$ as

$$
D=\widehat{D}^{(0)}+\gamma \widehat{D}^{(1)}+\gamma^{2} \widehat{D}^{(2)}+\cdots,
$$

we can obtain the terms in the expansion using (A.86) when we take

$$
\left\{\begin{array}{l}
\xi_{1}(t)=-\frac{1}{2} \frac{\left(\sigma^{A}\right)^{2}}{F\left(r_{t}, t ; T\right)}\left(\widehat{D}_{y_{A}}^{(0)}\right)^{2}-\frac{1}{2} \frac{\left(\sigma^{I}\right)^{2}}{F\left(r_{t}, t ; T\right)}\left(\widehat{D}_{y_{I}}^{(0)}\right)^{2}-\rho \frac{\sigma^{A} \sigma^{I}}{F\left(r_{t}, t ; T\right)} \widehat{D}_{y_{A}}^{(0)} \widehat{D}_{y_{I}}^{(0)}, \\
\xi_{2}(t)=-\frac{\left(\sigma^{A}\right)^{2}}{F\left(r_{t}, t ; T\right)} \widehat{D}_{y_{A}}^{(0)} \widehat{D}_{y_{A}}^{(1)}-\frac{\left(\sigma^{I}\right)^{2}}{F\left(r_{t}, t ; T\right)} \widehat{D}_{y_{I}}^{(0)} \widehat{D}_{y_{I}}^{(1)}-\rho \frac{\sigma^{A} \sigma^{I}}{F\left(r_{t}, t ; T\right)}\left(\widehat{D}_{y_{A}}^{(0)} \widehat{D}_{y_{I}}^{(1)}+\widehat{D}_{y_{I}}^{(0)} \widehat{D}_{y_{A}}^{(1)}\right) .
\end{array}\right.
$$

As in the single cohort case, we can approximate solutions to (12) and (41) when $\gamma>0$ is small. As usual, we write out explicitly the dependence of $\widehat{D}^{(0)}$ and $\widehat{D}^{(1)}$ on $\alpha$ :

$$
\widehat{D}^{(0)}=: \widehat{D}^{(0,0)}+\alpha \widehat{D}^{(0,1)}, \quad \widehat{D}^{(1)}=: \widehat{D}^{(1,0)}+\alpha \widehat{D}^{(1,1)}+\alpha^{2} \widehat{D}^{(1,2)} \text {. }
$$

Similar to (38) we then obtain

$$
\left\{\begin{aligned}
\widehat{D}^{(0,0)}\left(y^{I}, r, S^{I}, t\right) & =\mathbb{E}_{t}^{\mathbb{Q}}\left[\int_{t}^{T} \mathrm{e}^{-\int_{t}^{s} r_{u} d u}\left(\beta I \Lambda^{I}\left(u, Y_{u}^{I}\right) S_{u}^{I}-\beta \bar{p}_{I} S_{u}^{I}\right) d u\right], \\
\widehat{D}^{(0,1)}\left(y^{A}, r, S^{A}, t\right) & =\mathbb{E}_{t}^{\mathbb{Q}}\left[\int_{t}^{T} \mathrm{e}^{-\int_{t}^{s} r_{u} d u} A S_{u}^{A} d u\right], \\
\widehat{D}^{(1,1)}\left(y^{I}, y^{A}, r, S^{I}, S^{A}, t\right) & =\rho \cdot \mathbb{E}_{t}^{\mathbb{Q}}\left[\int_{t}^{T} \mathrm{e}^{-\int_{t}^{u} r_{s} d s} \frac{\sigma^{I} \sigma^{A}}{F\left(r_{u}, u ; T\right)} \widehat{D}_{y_{I}}^{(0,0)} \widehat{D}_{y_{A}}^{(0,1)} d u\right], \\
\widehat{D}^{(1,2)}\left(y^{A}, r, S^{A}, t\right) & =\mathbb{E}_{t}^{\mathbb{Q}}\left[\int_{t}^{T} \mathrm{e}^{-\int_{t}^{u} r_{s} d s} \frac{1}{2} \frac{\left(\sigma^{A}\right)^{2}}{F\left(r_{u}, u ; T\right)}\left(\widehat{D}_{y_{A}}^{(0,1)}\right)^{2} d u\right] .
\end{aligned}\right.
$$

Now we can use (39) and (44) after replacing $\widehat{H}$ by $\widehat{D}$ to obtain approximate solutions to (12) and (41) for small values of $\gamma$. Again we notice that the only difference is the $\widehat{D}^{(1,1)}$ term 
which is linear in $\rho$. In the numerical example we obtain $\alpha^{\gamma}(\rho)=\frac{p-\widehat{D}^{(0,1)}}{0.0354 \cdot 10^{9} \cdot \gamma}+0.020 \rho$. In a efficient competitive market one expects that the annuity factor is close to the risk-neutral price $p \simeq \widehat{D}^{(0,1)}$ so that $\alpha^{\gamma}$ is essentially proportional to $\rho$.

\section{Conclusion}

In this paper we have studied valuation of portfolios of mortality-contingent claims in a framework of systematic mortality risk and stochastic interest rates. We have focused on the dynamic exponential premium principle which captures in a non-linear way both the internal cross-hedge of the portfolio and the risk-aversion of the insurer. While the latter is hard to estimate precisely, we find that the resulting optimal quantities of annuities to sell are insensitive to risk-aversion parameter $\gamma$ indicating that the method is stable.

Our discussion has been centered around the "natural hedging" between life insurance and annuities, a setting also explored by Cox and Lin (2007). However, the developed tools are useful for any general portfolio of insurance claims, since any difference in contracts will allow for some degree of cross-hedging. In that sense, our main contribution is a general framework for treating dynamic hedging of mortality risk portfolios. In the same vein, the asymptotic expansions derived in Section 6 should be interpreted as the effects of model parameters (quantity $\alpha$, stochastic mortality timescale $\delta$ and risk-aversion parameter $\gamma$ ) on contract premia. For instance, a practitioner interested in modifying her static pricing rule to correct for stochastic mortality would benefit from seeing the $\delta$-expansion in Section 6.2 that shows that the correction terms are related to expectations under certain risk-neutral measures.

We have also obtained two premium principles that are "semi-linear". The instantaneous Sharpe ratio rule $\mathcal{H}$ is a special case of good deal bounds. It is only sensitive to large internal cross-hedges (those that modify the sign of $\mathcal{H}_{y}$ ). Unfortunately, this feature is limited by lack of simple rules on the nonlinearity of (29) which depends on a non-trivial manner on the parameters of cashflow function $f$. The relative indifference measure $\hat{\mathbb{Q}}$ involves a nonlinear Girsanov

transformation that uses the base valuation $H^{\beta I}$. It incorporates the internal cross-hedge and is used as a building block of the $\alpha$-expansion in Section 6.1.

Finally, we have also studied extensions that take into account the age structure of mortality rates. We find that the correlation $\rho$ between annuity and life insurance pools has a first-order effect on valuation, with the optimal quantity to sell $\alpha^{*}$ essentially proportional to $\rho$ for efficient market prices. This can be used to simplify practical applications by prorating all single-pool answers with respect to $\rho$.

\section{Acknowledgment}

This project has been supported by a CKER Research Grant administered by the SOA. We also thank Jenny Young for many useful discussions. 


\section{Appendix: Proofs of Analytical Results}

\section{Proof of Theorem 3.1}

Proof: Using the dynamic programming principle, it can be shown that the value function $V$ is the unique viscosity solution of the following Hamilton-Jacobi-Bellman (HJB) equation (see e.g. Duffie and Zariphopoulou (1993), Theorems 4.1 or 4.2; Bayraktar and Young (2007b), Propositions 3.3 and 3.6):

$$
\left\{\begin{array}{l}
V_{t}+r x V_{x}+\left(a_{0} r+b_{0}\right) V_{r}+\frac{1}{2}(c r+d) V_{r r} \\
+\max _{\pi}\left[-C\left(t, T_{1}\right)(\Delta a r+\Delta b) \pi V_{x}+\frac{1}{2} C\left(t, T_{1}\right)^{2}(c r+d) \pi^{2} V_{x x}-C\left(t, T_{1}\right)(c r+d) \pi V_{x r}\right]=0 \\
V(x, r, T)=-\mathrm{e}^{-\gamma x} .
\end{array}\right.
$$

Let us denote the generators of the $r$ processes by

$$
\mathcal{L}_{r}^{\mathbb{P}}=\left(a_{0} r+b_{0}\right) \partial_{r}+\frac{1}{2}(c r+d) \partial_{r r}^{2}, \quad \mathcal{L}_{r}^{\mathbb{Q}}=(a r+b) \partial_{r}+\frac{1}{2}(c r+d) \partial_{r r}^{2} .
$$

It follows that the optimal $\pi^{V}$ is obtained in feedback form as the unique maximizer of the Hamiltonian term in (A.53) and one may write (A.53) in the shorter form as

$$
V_{t}+r x V_{x}+\mathcal{L}_{r}^{\mathbb{P}} V-\frac{\left[(\Delta a r+\Delta b) V_{x}+(c r+d) V_{x r}\right]^{2}}{2(c r+d) V_{x x}}=0 .
$$

Note that the value function $V$ does not depend on $T_{1}$. A straightforward calculation shows that $V$ may be factored as stated, with $\psi$ solving (14). The smoothness of $\psi$ (see Krylov (1980), Theorem 2.9.10) together with (13) implies that the value function indeed is the smooth solution of (A.53).

\section{Proof of Theorem 3.2}

Proof: Using dynamic programming principle again it follows that $U$ is the unique viscosity solution of

$$
\left\{\begin{aligned}
U_{t} & +r x U_{x}-f \cdot U_{x}+\mathcal{L}_{r}^{\mathbb{P}} U+\mathcal{L}_{Y} U-\Lambda(t, y) S U_{S}+ \\
& +\max _{\pi}\left[-C(\Delta a r+\Delta b) \pi U_{x}+\frac{1}{2} C^{2}(c r+d) \pi^{2} U_{x x}-C(c r+d) \pi U_{x r}\right]=0 \\
U(x, y, r, S, T) & =-\mathrm{e}^{-\gamma x}
\end{aligned}\right.
$$

in which $\mathcal{L}_{Y} \triangleq \mu \partial_{y}+\frac{1}{2} \sigma^{2} \partial_{y y}$ is the infinitesimal generator of (4). Based on properties of exponential utility, we expect that the wealth $w$ will factor out and therefore posit that

$$
U(x, y, r, S, t) \equiv V(x, r, t) \phi(y, r, S, t) .
$$


Maximizing the Hamiltonian term with $\pi$ on the second line of (A.55) yields

$$
\pi^{U}=-\frac{F(r, t ; T)}{\gamma C\left(t, T_{1}\right)} \frac{\Delta a r+\Delta b}{c r+d}+\frac{V_{x} \phi_{r}+V_{x r} \phi}{C\left(t, T_{1}\right) V_{x x} \phi} .
$$

Plugging this into the ansatz (A.56) and separating the $V$ and $\phi$ terms produces

$$
\begin{aligned}
V\left[\phi_{t}+\mathcal{L}_{r}^{\mathbb{P}} \phi+\mathcal{L}_{Y} \phi\right. & \left.-\Lambda(t, y) S \phi_{s}\right]+\phi\left(V_{t}+r x V_{x}+f \frac{\gamma}{F} V+\mathcal{L}_{r}^{\mathbb{P}} V\right) \\
& +V_{r} \phi_{r}(c r+d)-\frac{\left[(\Delta a r+\Delta b) V_{x} \phi+(c r+d)\left[V_{x r} \phi+V_{x} \phi_{r}\right]\right]^{2}}{2(c r+d) V_{x x} \phi}=0 .
\end{aligned}
$$

Making use of (A.54) and simplifying leaves us with

$$
\left\{\begin{array}{r}
\phi_{t}+\mathcal{L}_{r}^{\mathbb{Q}} \phi+\mathcal{L}_{Y} \phi-\Lambda(t, y) S \phi_{S}+\frac{\gamma}{F(r, t ; T)} f \phi-\frac{c r+d}{2} \frac{\phi_{r}^{2}}{\phi}=0 \\
\phi(y, r, S, T)=1 .
\end{array}\right.
$$

Finally, setting $L(y, r, S, t) \triangleq \frac{1}{\gamma} \ln \phi(y, r, S, t)$ yields (18).

\section{Proof of Theorem 3.3}

\section{ProOF:}

We will use Theorem 6.6.2 of Fleming and Rishel (1975) and some arguments from the proof of Lemma 4.1 in Pham (2002) to prove our result.

Making the substitution in (19) and simplifying using

$$
\left\{\begin{aligned}
F_{t}+(a r+b) F_{r}+\frac{1}{2}(c r+d) F_{r r}-r F & =0 \\
F\left(r, T_{1} ; T_{1}\right) & =1 .
\end{aligned}\right.
$$

we obtain the following partial differential equation for $H$ :

$$
\left\{\begin{array}{r}
H_{t}+\mathcal{L}_{r}^{\mathbb{Q}} H+\mu H_{y}+\frac{1}{2} \sigma^{2}\left(H_{y y}+\frac{\gamma}{F(r, t ; T)} H_{y}^{2}\right)-\Lambda(t, y) S \cdot H_{S}-r H+f=0 \\
H(y, r, S, T)=0
\end{array}\right.
$$

Let us denote the Legendre transform of $K$ by

$$
E(q ; r, y, t) \triangleq \max _{p \in \mathbb{R}}\{q \cdot p-K(p)\}=\frac{1}{2} q^{2} \frac{F(r, t ; T)}{\gamma \sigma^{2}} .
$$

Observe that the first term in $(21)$ is simply $E\left(q_{u}\right)$. We have a duality relationship between $K$ and $E$,

$$
K(p)=\max _{q \in \mathbb{R}}\{q \cdot p-E(q)\}
$$


Next, we introduce a change of variables to make the coefficients of the second order derivative terms in (A.59) bounded (since the domain of the pde is unbounded we need these coefficients bounded):

$$
\tilde{r}=g(r) \triangleq \frac{2}{c} \sqrt{c r+d}, \quad \mathcal{L}_{\tilde{r}}=\left(\frac{a g^{-1}(\tilde{r})+b-(1 / 4) c}{\sqrt{c \tilde{r}+d}}\right) \partial_{\tilde{r}}+\frac{1}{2} \partial_{\tilde{r} \tilde{r}},
$$

where $g^{-1}$ is the functional inverse of $g$. Then defining $R(y, \tilde{r}, S, t)=H(y, r, S, t)$, the pde for $R$ becomes

$$
R_{t}+\mathcal{L}_{\tilde{r}} R+\mathcal{L}_{Y} R+\max _{q \in \mathbb{R}}\left(q R_{y}-\tilde{E}(q)\right)-\Lambda(t, y) S \cdot R_{S}-r \cdot R+f=0
$$

where $\tilde{E}(q ; y, \tilde{r}, t)=E(q ; y, r, t)=\frac{1}{2} q^{2} \frac{F\left(g^{-1}(\tilde{r}), t ; T\right)}{\gamma \sigma^{2}}$.

It is enough to prove that (A.61) has a unique classical solution. Under our assumptions, in (A.61) the coefficients of the first-order derivatives have bounded derivatives, and the coefficients of the second-order derivatives are all bounded. The only problematic term in the pde is the nonlinear term from the maximization $\max _{q \in \mathbb{R}}$. Specifically, the difficulty is that this maximization is over an unbounded domain. To circumvent this, let us instead consider the following family of differential equations, indexed by $k$,

$$
R_{t}^{(k)}+\mathcal{L}_{\tilde{r}} R^{(k)}+\mathcal{L}_{Y} R^{(k)}+\max _{q \in B_{k}}\left\{q R_{y}^{(k)}-\tilde{E}(q)\right\}-\Lambda(t, y) S \cdot R_{S}^{(k)}-r R^{(k)}+f=0,
$$

in which $B_{k}=\{q \in \mathbb{R}:|q| \leq k\}, k>0$ is a ball of radius $k$. Now, Theorem 6.6.2 of Fleming and Rishel (1975) allows us to conclude that for each $k>0$, the pde (A.62) has a unique classical solution which moreover satisfies a polynomial growth condition.

Applying the standard verification theorem (see Fleming and Soner (1993) Theorem 3.1) we have that the solution of (A.62) can be written as

$R^{(k)}(y, \tilde{r}, S, t)=\sup _{q \in B_{k}} \mathbb{E}^{Q^{q}}\left[\int_{t}^{T} \mathrm{e}^{-\int_{t}^{u} r_{s} d s}\left(-\tilde{E}\left(q_{u}\right)+f\left(\Lambda\left(u, Y_{u}\right), S_{u}\right)\right) d u \mid Y_{t}=y, \tilde{r}_{t}=\tilde{r}, S_{t}=S\right]$,

where under the probability measure $Q^{q}$ the dynamics of the process $Y$ are given by (22). Using this control representation, we will show that the derivative $R_{y}^{(k)}$ is bounded from above and below, and

$$
\begin{aligned}
& \left|\frac{R^{(k)}(y+\varepsilon, \tilde{r}, S, t)-R^{(k)}(y, \tilde{r}, S, t)}{\varepsilon}\right| \\
& \leq \sup _{q \in B_{k}} \mathbb{E}^{Q^{q}}\left[\int_{t}^{T} \mathrm{e}^{-\int_{t}^{u} r_{s} d s} \frac{\left|f\left(\Lambda\left(Y_{u}^{t, y+\varepsilon}, u\right), S_{u}\right)-f\left(\Lambda\left(Y_{u}^{t, y}, u\right), S_{u}\right)\right|}{\varepsilon} \mid \tilde{r}_{t}=\tilde{r}, S_{t}=S\right] \leq C,
\end{aligned}
$$

for some $C \geq 0$ that does not depend on $k$, in which $Y^{t, y}$ is the process $Y$ started at $y$ at time $t$. The second inequality follows since $f$ is linear, $\Lambda$ has a bounded derivative, and $Y$ is Lipschitz in its initial condition (which can be proved by Gronwall's lemma), i.e.

$$
\left|Y_{u}^{t, y}-Y_{u}^{t, z}\right| \leq C_{1}|y-z|, \quad t \leq u \leq T, y, z \in \mathbb{R},
$$

in which the constant $C_{1} \geq 0$ does not depend on $q$. 
Now, for a large enough and arbitrary constant $C_{2} \geq 0$, the function $q \rightarrow q R_{y}^{(k)}-\tilde{E}(q)$ attains its maximum at

$$
\hat{q}^{(k)}=\frac{\gamma \sigma^{2} R_{y}^{(k)}}{F\left(g^{-1}(\tilde{r}), t ; T\right)} \in\left[-C_{3}, C_{3}\right], \quad \tilde{r} \leq C_{2},
$$

for some constant $C_{3} \geq 0$ that does not depend on $k$. Therefore, for $k \geq C_{3}$, we have

$$
\max _{q \in B_{k}}\left\{q R_{y}^{(k)}-\tilde{E}(q)\right\}=\max _{q \in \mathbb{R}}\left\{q R_{y}^{(k)}-\tilde{E}(q)\right\},
$$

which implies that $R^{(k)}$ is a smooth solution of (A.62) on $(t, y, \tilde{r}, S) \in[0, T] \times \mathbb{R} \times\left[0, C_{2}\right] \times[0,1]$. Since $C_{2}$ is arbitrary, we conclude, as in the proof of Theorem 4.1 in Pham (2002), that (A.62) has a smooth solution with polynomial growth condition in $y$ and $\tilde{r}$.

Finally, let us show the uniqueness property of (A.62) among the solutions with polynomial growth. Assume that there are two classical solutions $R^{1}$ and $R^{2}$ to (A.62) and let $\bar{R}=R^{1}-R^{2}$. Then, $\bar{R}$ satisfies the linear partial differential equation

$$
\left\{\begin{array}{l}
\bar{R}_{t}+\alpha(\tilde{r}) \bar{R}_{\tilde{r}}+\frac{1}{2} \bar{R}_{\tilde{r} \tilde{r}}+\mu \bar{R}_{y}+\frac{1}{2} \sigma^{2} \bar{R}_{y y}+\frac{1}{2} \sigma^{2} \frac{\gamma}{F(r, t ; T)}\left(R_{y}^{1}+R_{y}^{2}\right) \cdot \bar{R}_{y}-\Lambda(t, y) S \bar{R}_{S}-r \bar{R}=0 \\
\bar{R}(y, \tilde{r}, S, T)=0 .
\end{array}\right.
$$

Since the terminal condition above is identically zero and there is no forcing term, using either Feynman-Kac theorem or the maximum principle (see Evans (1998) and Protter and Weinberger (1967)), it follows that $\bar{R} \equiv 0$.

\section{Proof of Theorem 3.4}

Proof: The argument is similar to that of the proof of Theorem 2.3 in Bayraktar and Young (2008): the derivative of the indifference price with respect to $S$ is a solution of the linear pde

$$
\mathcal{L}^{S} H_{S}=-f_{S}, \quad U(y, r, S, T)=0,
$$

in which

$$
\begin{aligned}
\mathcal{L}^{S} U & \triangleq U_{t}+(a r+b) U_{r}+\frac{1}{2}(c r+d) U_{r r}+\mu(y) U_{y}+\frac{1}{2} \sigma^{2} U_{y y} \\
& -\lambda(t, y) U-\lambda(t, y) S U_{S}+\sigma^{2} \frac{\gamma}{F} H_{y} U_{y}-r U
\end{aligned}
$$

Since $\mathcal{L}^{S} 0=0$ and $L^{S} H_{S}=-f_{S}$ (and the values of $H_{S}$ and the zero-function match at $T$ ), then it follows from the maximum principle for linear parabolic pdes (see e.g. Evans (1998)) that $\operatorname{sgn}\left(H_{S}\right)=\operatorname{sgn}\left(f_{S}\right)$. We can also argue this result using the representation in (20). Since $S_{t}$ increases in its initial value, and $f$ is an increasing function of $S$, we can immediately see that the right hand side in (20) increases when we choose $S_{t}=S$ to be a larger value.

Similarly, the derivative of the indifference price with respect to $r$ satisfies,

$$
\mathcal{L}^{r} H_{r}=\frac{1}{2} \gamma \sigma^{2} \frac{F_{r}}{F^{2}} H_{y}^{2}, \quad H_{r}(y, r, S, T)=0,
$$

for a linear parabolic operator $\mathcal{L}^{r}$. Since $\mathcal{L}^{r} 0=0$ and $F_{r}<0$, we have that $\mathcal{L}^{r} H_{r} \leq \mathcal{L}^{r} 0=0$, which implies that $H_{r} \geq 0$. 
The behavior of the $Y$-derivatives of $H$ follow from the representation of the exponential indifference premium in (21). First, as $Y_{t}=y$ increases, so does $Y_{u}, u \in(t, T]$. Consequently, by the theorem hypothesis $f\left(\Lambda\left(u, Y_{u}\right)\right)$ increases and therefore $H_{y} \geq 0$. Similarly, it can be seen that $H$ is convex in $y$. Recall that $Y_{u}$ is linear in its initial value $Y_{t}=y$. Since both $y \rightarrow \Lambda(t, y)$ and $\lambda \rightarrow f(\lambda, t)$ are convex, the convexity of $H$ in $y$ follows as a result of the properties of the supremum and (21). But we know that $H$ is two times differentiable in $y$ as a result of Theorem 3.3 , which implies that $H_{y y} \geq 0$.

\section{Proof of Theorem 3.5}

Proof: Since $\Lambda$ in (21) is an increasing and convex function of its second variable, and $Y$ is linear in its initial condition, we observe from (21) that the indifference price $H$ is increasing and convex in $\alpha$. Also recall from Theorem 3.4 that $H_{y} \geq 0$.

Let $\mu_{1}(y, t) \leq \mu_{2}(y, t)$. Let us denote the solution of $((\mathrm{A} .59))$ when $\mu$ is replaced by $\mu_{1}$ and $\mu_{2}$ by $H^{(1)}$ and $H^{(2)}$ respectively. The indifference price $H^{(1)}$ satisfies

$$
\mathcal{L}^{(1)} H^{(1)} \triangleq H_{t}+\mathcal{L}_{r}^{\mathbb{Q}} H+\mu_{1} H_{y}+\frac{1}{2} \sigma^{2}\left(H_{y y}+\frac{\gamma}{F(r, t ; T)} H_{y}^{2}\right)-\Lambda(t, y) S \cdot H_{s}-r H+f=0 .
$$

On the other hand since $H_{y}^{(2)} \geq 0$ we have

$$
\mathcal{L}^{(1)} H^{(2)}=\left(\mu_{1}-\mu_{2}\right) H_{y}^{(2)} \leq 0
$$

which shows that $H^{(2)}$ is a super-solution of $((\mathrm{A} .59))$ when $\mu$ is replaced by $\mu_{1}$. Therefore, $H^{(2)} \geq H^{(1)}$.

A similar proof can be carried out for the second part of the statement using $H_{y y} \geq 0$.

\section{Proof of Theorem 3.6}

Proof: We have $\pi^{f}=\pi^{U}-\pi^{V}$ since this difference precisely captures the change in optimal investment by the insurer brought about by selling $f$. Recall that $\pi^{U}$ and $\pi^{V}$ are obtained by maximizing the Hamiltonian terms in (A.55) and (A.53) respectively. Expanding the expression already computed in (A.57) gives

$$
\pi_{t}^{U}=\frac{F(r, t ; T)}{\gamma C\left(t, T_{1}\right)}\left[-\frac{\Delta a r+\Delta b}{c r+d}-C(t, T)-\psi_{r}(\cdot, t)\right]+\frac{1}{C\left(t, T_{1}\right)}\left[C(t, T) X_{t}^{f}-\frac{\phi_{r}(\cdot, t)}{\phi(\cdot, t)}\right] .
$$

In the absence of claims, the optimal investment $\pi^{V}(x, r, t)$ in the $T$-bond, $\pi^{V}$ is given by (note the appearance of both $C\left(t, T_{1}\right)$ and $\left.C(t, T)\right)$

$$
\begin{aligned}
\pi^{V}(x, r, t) & =\frac{1}{C\left(t, T_{1}\right)}\left[\frac{\Delta a r+\Delta b}{c r+d} \frac{V_{x}}{V_{x x}}+\frac{V_{x r}}{V_{x x}}\right] \\
& =\frac{F(r, t ; T)}{\gamma C\left(t, T_{1}\right)}\left[-\frac{\Delta a r+\Delta b}{c r+d}+\frac{\gamma}{F(r, t ; T)} C(t, T) x-\psi_{r}-C(t, T)\right] .
\end{aligned}
$$


Taking the difference we obtain (23).

\section{Proof of Theorem 3.7}

Proof: We make use of linear pdes related to (A.58). First, the function $\phi$ which solves (A.58) is a sub-solution of

$$
\left\{\begin{aligned}
\underline{J}_{t}+\mathcal{L}_{r}^{\mathbb{Q}} \underline{J}+\mathcal{L}_{Y} \underline{J}-\Lambda(t, y) S \underline{J}_{s}+\frac{\gamma}{F(r, t ; T)} f \underline{J} & =0, \\
\underline{J}(y, r, S, T) & =1,
\end{aligned}\right.
$$

we have the upper bound (applying Feynman-Kac to $\underline{J}$ ):

$$
\phi(y, r, S, t) \leq \mathbb{E}_{t}^{\mathbb{Q}}\left[\exp \left(\int_{t}^{T} \frac{\gamma}{F} f d u\right)\right] .
$$

On the other hand $\phi$ of (A.58) is a super-solution of

$$
\left\{\begin{array}{r}
\bar{J}_{t}+\mathcal{L}_{r}^{\mathbb{Q}} \bar{J}+\mathcal{L}_{Y} \bar{J}-\Lambda(t, y) S \bar{J}_{S}+\frac{\gamma}{F(r, t ; T)} f \bar{J}-\frac{c r+d}{2} \frac{\bar{J}_{r}^{2}}{\bar{J}}-\frac{1}{2} \sigma^{2} \frac{\bar{J}_{y}^{2}}{\bar{J}}=0 \\
\bar{J}(y, r, S, T)=1
\end{array}\right.
$$

Using an exponential transformation $\bar{J}=\mathrm{e}^{\widehat{J}}$, we obtain

$$
\left\{\begin{aligned}
\widehat{J}_{t}+\mathcal{L}_{r}^{\mathbb{Q}} \widehat{J}+\mathcal{L}_{Y} \widehat{J}-\Lambda(t, y) S \widehat{J}_{S}+\frac{\gamma}{F(r, t ; T)} f=0 \\
\widehat{J}(y, r, S, T)=0
\end{aligned}\right.
$$

which leads to

$$
\widehat{J}(y, r, S, t)=\mathbb{E}_{t}^{\mathbb{Q}}\left[\int_{t}^{T} \frac{\gamma}{F(r, u ; T)} f d u\right]
$$

Combining (19), (A.68) and (A.69) we have (26).

Proof of Theorem 4.1 Proof: Let $\mathcal{H}\left(Y_{t}, r_{t}, S_{t}, t\right)$ be the value of obligation to pay at instantaneous rate $f$. To derive the pde satisfied by $\mathcal{H}$ we suppose that the insurer creates a portfolio $\Pi=\left(\Pi_{t}\right)_{t \geq 0}$ of this obligation and a self-financing trading strategy whose value at time $\mathrm{t}$ is $\left(V_{t}\right)_{t \geq 0}$ and which is composed of $\pi_{t} T_{1}$-bonds and a money market account. Thus $\Pi_{t}=-\mathcal{H}_{t}+V_{t}$ with a continuous outflow of money at rate $f\left(\Lambda\left(t, Y_{t}\right), S_{t}\right)$.

Applying Îto's lemma to the smooth function $\mathcal{H}\left(Y_{t}, r_{t}, S_{t}, t\right)$, we obtain its dynamics as

$$
\begin{aligned}
d \mathcal{H}\left(Y_{t}, r_{t}, S_{t}, t\right) & =\mathcal{H}_{t} d t+\mathcal{H}_{r} d r_{t}+\frac{1}{2} \mathcal{H}_{r r} d[r, r]_{t}+\mathcal{H}_{y} d Y_{t}+\frac{1}{2} \mathcal{H}_{y y} d[Y, Y]_{t}+\mathcal{H}_{S} d S_{t} \\
& =\mathcal{H}_{t} d t+\mathcal{H}_{r}\left[\left(a_{0} r_{t}+b_{0}\right) d t+\sqrt{c r_{t}+d} d W_{t}^{r}\right]+\frac{1}{2} \mathcal{H}_{r r}\left(c r_{t}+d\right) d t \\
& +\mathcal{H}_{y}\left(\mu\left(Y_{t}\right) d t+\sigma d W_{t}\right)+\frac{1}{2} \mathcal{H}_{y y} \sigma^{2} d t+\mathcal{H}_{S} \Lambda\left(t, Y_{t}\right) S_{t} d t
\end{aligned}
$$


Because $V_{t}$ is self-financing

$d V_{t}=\pi_{t} d F\left(r_{t}, t ; T_{1}\right)+r_{t}\left(V_{t}-\pi_{t} F\left(r_{t}, t ; T_{1}\right)\right) d t=\left[\pi_{t}\left(\Delta a r_{t}+\Delta b\right) F_{r}+r_{t} V_{t}\right] d t+\pi_{t} \sqrt{c r_{t}+d_{t}} F_{r} d W_{t}^{r}$.

It follows from (A.70) and (A.71) that

$$
\begin{aligned}
& \Pi_{t+h}=\Pi_{t}-\int_{t}^{t+h} d \mathcal{H}\left(Y_{u}, r_{u}, S_{u}, u\right)+\int_{t}^{t+h} d V_{s}-\int_{t}^{t+h} f\left(\Lambda\left(u, Y_{u}\right), S_{u}\right) d s \\
& =\Pi_{t}-\int_{t}^{t+h}\left[\left(\mathcal{L}_{H}-r_{u}\right) \mathcal{H}\left(Y_{u}, r_{u}, S_{u}, u\right)+f\left(\Lambda\left(u, Y_{u}\right), S_{u}\right)\right] d u \\
& +\int_{t}^{t+h} \sqrt{c r_{u}+d}\left(\pi_{u} F_{r}\left(r_{u}, u ; T_{1}\right)-\mathcal{H}_{r}\left(Y_{u}, r_{u}, S_{u}, u\right)\right) d W_{u}^{r}-\int_{t}^{t+h} \sigma \mathcal{H}_{\lambda}\left(Y_{u}, r_{u}, S_{u}, u\right) d W_{s} \\
& +\int_{t}^{t+h} \mathcal{H}_{S} \Lambda\left(u, Y_{u}\right) S_{u} d u+\int_{t}^{t+h} \pi_{s}\left(\Delta a r_{u}+\Delta b\right) F_{r}\left(r_{u}, u ; T\right) d u+\int_{t}^{t+h} r_{u} \Pi_{u} d u,
\end{aligned}
$$

in which $\mathcal{L}_{H}^{0}$ is as in (16) when $a$ and $b$ are replaced by $a_{0}$ and $b^{0}$ respectively. We now choose the number of $T_{1}$-bonds $\pi_{t}$ to minimize the local variance of the portfolio $\Pi$. To calculate $\pi$ in terms of $\mathcal{H}$ and finally obtain a pde that $\mathcal{H}$ satisfies, we need to calculate the conditional expectation and the variance of $\Pi_{t}$ given the information available at time $t$, which we denote by $\mathcal{F}_{t}$. The minimizer of the local variance

$$
\begin{aligned}
\lim _{h \rightarrow 0} \frac{1}{h} \operatorname{Var}\left(\Pi_{t+h} \mid \mathcal{F}_{t}\right) & =\lim _{h \rightarrow 0} \frac{1}{h} \mathbb{E}\left[\Pi_{t+h}-\mathbb{E}\left[\Pi_{t+h}^{2} \mid \mathcal{F}_{t}\right] \mid \mathcal{F}_{t}\right] \\
& =\left(c r_{t}+d\right)\left(\pi_{t} F_{r}\left(r_{t}, t ; T_{1}\right)-\mathcal{H}_{r}\left(Y_{t}, r_{t}, S_{t}, t\right)\right)^{2}+\sigma^{2} \mathcal{H}_{y}^{2}\left(Y_{t}, r_{t}, S_{t}, t\right),
\end{aligned}
$$

is given by

$$
\pi_{t}^{S R}=\frac{\mathcal{H}_{r}\left(Y_{t}, r_{t}, S_{t}, t\right)}{F_{r}\left(r_{t}, t ; T_{1}\right)}=-\frac{\mathcal{H}_{r}\left(Y_{t}, r_{t}, S_{t}, t\right)}{C\left(t, T_{1}\right) F\left(r_{t}, t ; T_{1}\right)} .
$$

Hence the minimum local variance of portfolio $\Pi$ at time $t$ is $\sigma^{2} \mathcal{H}_{y}^{2}\left(Y_{t}, r_{t}, S_{t}, t\right)$. The expected instantaneous change of the minimum variance portfolio becomes

$$
\lim _{h \rightarrow 0} \frac{1}{h}\left(\mathbb{E}\left[\Pi_{t+h} \mid \mathcal{F}_{t}\right]-\Pi_{t}\right)=-\left[\left(\mathcal{L}_{H}-r_{t}\right) \mathcal{H}\left(Y_{t}, r_{t}, S_{t}, t\right)+f\left(\Lambda\left(t, Y_{t}\right), S_{t}\right)\right]+r_{t} \Pi_{t},
$$

in which $\mathcal{L}_{H}$ is as in (16). Finally, to determine the equation satisfied by $\mathcal{H}$, we set expected instantaneous change of the portfolio equal to the short rate times the value of the portfolio plus $\gamma^{S R}$ times the local standard deviation of the portfolio:

$$
\lim _{h \rightarrow 0} \frac{1}{h}\left(\mathbb{E}\left[\Pi_{t+h} \mid \mathcal{F}_{t}\right]-\Pi_{t}\right)=\left(r_{t} \Pi_{t}+\gamma^{S R} \sqrt{\sigma^{2} \mathcal{H}_{y}^{2}\left(Y_{t}, r_{t}, S_{t}, t\right)}\right)
$$

The constant $\gamma^{S R}$ is the instantaneous Sharpe ratio demanded by the insurer and reflects the insurer's attitude towards risk. Higher $\gamma^{S R}$ means that the insurer demands more compensation (in terms of return on $\Pi$ ) for the risk she takes on. Plugging-in this into (A.75) and combining with (A.74), it follows that $\mathcal{H}=\mathcal{H}(y, r, s, t)$ solves $(27)$. The existence and uniqueness of similar pdes were studied by Bayraktar and Young (2008). Note that the first equation in (27) can also be written as

$$
\mathcal{H}_{t}+\mathcal{L}_{H} \mathcal{H}+\sup _{|q| \leq \gamma^{S R}}\left(q \sigma \mathcal{H}_{y}\right)-r_{t} \mathcal{H}+f\left(\Lambda\left(t, Y_{t}\right), Y_{t}\right)=0
$$

which immediately leads to (28). 


\section{Proof of Theorem 6.1}

Proof: We carry out an asymptotic expansion of $H^{\alpha A \mid \beta I}$ in terms of $\alpha$, the quantity of annuities sold. Namely we write

$$
H^{\alpha A+\beta I} \triangleq H^{(0)}+\alpha H^{(1)}+\alpha^{2} H^{(2)}+\alpha^{3} H^{(3)}+o\left(\alpha^{3}\right) .
$$

Substituting this into (A.59) we find that:

$$
H_{t}^{(0)}+\mathcal{L}_{H} H^{(0)}+\frac{1}{2} \sigma^{2} \frac{\gamma}{F\left(r_{t}, t ; T\right)}\left(H_{y}^{(0)}\right)^{2}-r H^{(0)}=-\beta \lambda_{t} S_{t}+\beta \bar{p}_{I} S_{t}, \quad H^{(0)}(y, r, S, T)=0 .
$$

Comparing with (A.59) we see that $H^{(0)} \equiv H^{\beta I}$ is the indifference price of a contract paying out $\beta\left(\Lambda\left(t, Y_{t}\right) S_{t}-\bar{p}_{I} S_{t}\right)$, i.e. of $\beta$ life-insurance contracts. Next,

$$
H_{t}^{(1)}+\mathcal{L}_{H} H^{(1)}-r H^{(1)}=-A S_{t}-\sigma^{2} \frac{\gamma}{F\left(r_{t}, t ; T\right)} H_{y}^{(1)} H_{y}^{(0)}, \quad H^{(1)}(y, r, S, T)=0 .
$$

Proceeding with the expansion,

$$
\begin{aligned}
& H_{t}^{(2)}+\mathcal{L}_{H} H^{(2)}-r H^{(2)}=-\frac{1}{2} \sigma^{2} \frac{\gamma}{F\left(r_{t}, t ; T\right)}\left[2 H_{y}^{(0)} H_{y}^{(2)}+\left(H_{y}^{(1)}\right)^{2}\right], \quad H^{(2)}(y, r, S, T)=0 \\
& H_{t}^{(3)}+\mathcal{L}_{H} H^{(3)}-r H^{(3)}=-\sigma^{2} \frac{\gamma}{F\left(r_{t}, t ; T\right)}\left[H_{y}^{(0)} H_{y}^{(3)}+H_{y}^{(1)} H_{y}^{(2)}\right], \quad H^{(3)}(y, r, S, T)=0 .
\end{aligned}
$$

We observe that (A.77)-(A.78) are linear and therefore will have a Feynman-Kac representation (Karatzas and Shreve 1991). Indeed, define the measure $\hat{\mathbb{Q}}$ as stated in the theorem. Then by a Girsanov change of measure, under $\hat{\mathbb{Q}}$ the drift of $Y$ is $\mu+\sigma^{2} \frac{\gamma}{F} H_{y}^{(0)}$. Finally, applying the Feynman-Kac formula to the linear pdes above, we obtain that $H^{(i)}, i=1,2,3$ have the representation given in the theorem.

In terms of the problem faced by a price-taker, we need to compute

$$
\arg \max _{\alpha}\left\{\alpha A p-H^{\alpha A \mid \beta I}\right\}=\arg \max _{\alpha}\left\{\alpha\left(A p-H^{(1)}\right)-\alpha^{2} H^{(2)}+o\left(\alpha^{2}\right)\right\} .
$$

We see that if $A p-H^{(1)}$ is small, then the optimal $\alpha$ is small and the expansion above implies $\alpha^{*} \simeq \frac{A p-H^{(1)}(y, r, S, t)}{2 H^{(2)}(y, r, S, t)}$, as in $(33)$.

\section{Proof of Theorem 6.2}

Proof: Using (34), we first re-write (A.59) as

$$
H_{t}+\mathcal{L}_{r}^{\mathbb{Q}} H+\delta \mu H_{y}+\frac{1}{2} \delta \sigma^{2}\left(H_{y y}+\frac{\gamma}{F\left(r_{t}, t ; T\right)} H_{y}^{2}\right)-\Lambda(t, y) S \cdot H_{S}-r H+f=0,
$$

with $H(y, r, S, T)=0$. Next we perform an expansion in powers of $\delta$,

$$
H=\widetilde{H}^{(0)}+\delta \widetilde{H}^{(1)}+\cdots
$$


Substituting this expansion in (A.79) and collecting the $\mathcal{O}(1)$-terms we obtain the linear pde

$$
\widetilde{H}_{t}^{(0)}+\mathcal{L}_{r}^{\mathbb{Q}} \widetilde{H}^{(0)}-\Lambda(t, y) S \cdot \widetilde{H}_{S}^{(0)}-r H^{(0)}=-f,
$$

with $\widetilde{H}^{(0)}(y, r, S, T)=0$ as usual. The solution of (A.81) is given by

$$
\widetilde{H}^{(0)}(y, r, S, t)=\int_{t}^{T} F(r, t ; u) f\left(\Lambda(u, y), \mathrm{e}^{-\int_{t}^{u} \Lambda(s, y) d s} \cdot S\right) d u,
$$

It is worth pointing out that $\widetilde{H}^{(0)}$ is also linear in $\alpha$. Inserting (A.80) into (A.79) and collecting $\mathcal{O}(\delta)$ terms together we obtain the linear pde that $\widetilde{H}^{(1)}$ solves

$$
\widetilde{H}_{t}^{(1)}+\mathcal{L}_{r}^{\mathbb{Q}} \widetilde{H}^{(1)}-\Lambda(t, y) S \widetilde{H}_{S}^{(1)}-r \widetilde{H}^{(1)}=-\mu \widetilde{H}_{y}^{(0)}-\frac{1}{2} \sigma^{2}\left(\widetilde{H}_{y y}^{(0)}+\frac{\gamma}{F\left(r_{t}, t ; T\right)}\left(\widetilde{H}_{y}^{(0)}\right)^{2}\right),
$$

with zero as the terminal condition. The solution of this linear pde can also be determined as

$$
\widetilde{H}^{(1)}(y, r, S, t)=\mathbb{E}^{\mathbb{Q}}\left[\int_{t}^{T} \mathrm{e}^{-\int_{t}^{u} r_{s} d s}\left\{\mu \widetilde{H}_{y}^{(0)}+\frac{1}{2} \sigma^{2}\left(\widetilde{H}_{y y}^{(0)}+\frac{\gamma}{F\left(r_{u}, u ; T\right)}\left(\widetilde{H}_{y}^{(0)}\right)^{2}\right)\right\} d u \mid r_{t}=r\right]
$$

in which the argument of the functions $\widetilde{H}_{y}^{(0)}$ and $\widetilde{H}_{y y}^{(0)}$ is $\left(r_{u}, y, S \exp (-\Lambda(t, y)(u-t))\right.$, $\left.u\right)$, with $r_{u}$ being the only stochastic variable. Note that $H^{(1)}$ is a second order polynomial in terms of $\alpha$.

Returning to $H^{\alpha A \mid \beta I}=H^{\alpha A+\beta I}-H^{\beta I}$ and applying the above expansion to each term on the right hand side, we have

$$
\begin{aligned}
H^{\alpha A \mid \beta I}(y, r, S, t) & ={ }^{0} \widetilde{H}^{\alpha A+\beta I}-{ }^{0} \widetilde{H}^{\beta I}+\delta\left({ }^{1} \widetilde{H}^{\alpha A+\beta I}-{ }^{1} \widetilde{H}^{\beta I}\right)+\mathcal{O}\left(\delta^{2}\right) \\
& \left.=\int_{t}^{T} F(r, t ; u) A \Lambda(u, y) \cdot \mathrm{e}^{-\int_{t}^{u} \Lambda(s, y) d s} \cdot S\right) d u+\delta^{1} \widetilde{H}^{\alpha A \mid \beta I}+\mathcal{O}\left(\delta^{2}\right),
\end{aligned}
$$

since the zero-order terms are linear in the payoff, and therefore additive. Above, the second term satisfies

$$
\begin{aligned}
{ }^{1} \widetilde{H}_{t}^{\alpha A \mid \beta I}+\mathcal{L}_{r}^{\mathbb{Q} 1} \widetilde{H}^{\alpha A \mid \beta I}-\Lambda(t, y) S^{1} \widetilde{H}_{S}^{\alpha A \mid \beta I}-r^{1} \widetilde{H}^{\alpha A \mid \beta I}=-\mu^{0} \widetilde{H}_{y}^{\alpha A} \\
-\frac{1}{2} \sigma^{2}\left({ }^{0} \widetilde{H}_{y y}^{\alpha A}+\frac{\gamma}{F\left(r_{t}, t ; T\right)}\left({ }^{0} \widetilde{H}_{y}^{\alpha A}\right)^{2}+\frac{2 \gamma}{F(r, t ; T)}{ }^{0} \widetilde{H}_{y}^{\alpha A} \cdot{ }^{0} \widetilde{H}_{y}^{\beta I}\right) .
\end{aligned}
$$

or just ${ }^{1} \widetilde{H}^{\alpha A \mid \beta I}=\alpha \widetilde{H}^{(1,1)}+\alpha^{2} \widetilde{H}^{(1,2)}$ again by linearity of all the respective pdes.

Next, we find an approximate value for the optimal $\alpha^{\delta}$ in (12) when $\delta>0$ is small, i.e., when the mortality rate changes at a very slow rate. To this end, we first explicitly show how the functions $\widetilde{H}^{(0)}$ and $\widetilde{H}^{(1)}$ depend on $\alpha$ (recall that $\widetilde{H}^{(0)}$ is linear in $\alpha$ and $\widetilde{H}^{(1)}$ is quadratic):

$$
\widetilde{H}^{(0)}=: \widetilde{H}^{(0,0)}+\alpha \widetilde{H}^{(0,1)}, \quad \widetilde{H}^{(1)}=: \widetilde{H}^{(1,0)}+\alpha \widetilde{H}^{(1,1)}+\alpha^{2} \widetilde{H}^{(1,2)},
$$

The functions $\widetilde{H}^{(0,0)}$ and $\widetilde{H}^{(0,1)}$ are explicitly given by

$$
\begin{aligned}
& \widetilde{H}^{(0,0)}(y, r, S, t)=\int_{t}^{T} F(r, t ; u) \beta\left(I \Lambda(u, y)-\bar{p}_{I}\right) \mathrm{e}^{-\int_{t}^{u} \Lambda(s, y) d s} \cdot S d u \\
& \widetilde{H}^{(0,1)}(y, r, S, t)=\int_{t}^{T} F(r, t ; u) A \mathrm{e}^{-\int_{t}^{u} \Lambda(s, y) d s} \cdot S d u,
\end{aligned}
$$


and represent the indifference price of $\beta$ life-insurances and one annuity respectively. The functions $\widetilde{H}^{(1, i)}, i \in\{0,1,2\}$ are in turn solutions of the linear pdes

$$
u_{t}+\mathcal{L}_{r}^{\mathbb{Q}} u-\Lambda(t, y) S u_{S}-r u=\xi_{i}, \quad u(y, r, S, T)=0,
$$

in which

$$
\left\{\begin{array}{l}
\xi_{0}(t)=-\mu \widetilde{H}_{y}^{(0,0)}-\frac{1}{2} \sigma^{2}\left(\widetilde{H}_{y y}^{(0,0)}+\frac{\gamma}{F\left(r_{t}, t ; T\right)}\left(\widetilde{H}_{y}^{(0,0)}\right)^{2}\right) \\
\xi_{1}(t)=-\mu \widetilde{H}_{y}^{(0,1)}-\frac{1}{2} \sigma^{2}\left(\widetilde{H}_{y y}^{(0,1)}+\frac{2 \gamma \widetilde{H}_{y}^{(0,0)} \widetilde{H}_{y}^{(0,1)}}{F\left(r_{t}, t ; T\right)}\right) \quad \xi_{2}(t)=-\frac{1}{2} \sigma^{2} \frac{\gamma}{F\left(r_{t}, t ; T\right)}\left(\widetilde{H}_{y}^{(0,1)}\right)^{2},
\end{array}\right.
$$

Applying Feynman-Kac formula, we recover (36). Now, an approximate value for the optimal $\alpha^{*}$ in (12) can be obtained as in (37).

\section{Proof of Theorem 6.3 and Corollary 6.1}

Proof: We start with the Taylor expansion

$$
H=\widehat{H}^{(0)}+\gamma \widehat{H}^{(1)}+\gamma^{2} \widehat{H}^{(2)}+\cdots .
$$

All the terms in this expansion satisfy linear pdes. In fact $\widehat{H}^{(i)}, i \in\{0,1,2\}$ are the solutions of

$$
u_{t}+\mathcal{L}_{H} u-r u=\xi_{i}, \quad u(y, r, S, T)=0,
$$

in which

$$
\xi_{0}(t)=-f\left(\Lambda\left(t, Y_{t}\right), S_{t}\right), \quad \xi_{1}(t)=-\frac{1}{2} \frac{\sigma^{2}}{F\left(r_{t}, t ; T\right)}\left(\widehat{H}_{y}^{(0)}\right)^{2}, \quad \xi_{2}(t)=-\frac{\sigma^{2}}{F\left(r_{t}, t ; T\right)} \widehat{H}_{y}^{(0)} \cdot \widehat{H}_{y}^{(1)} .
$$

Thus, by Feynman-Kac formula $\widehat{H}^{(i)}, i \in\{0,1,2\}$ can be explicitly written as

$$
\widehat{H}^{(i)}(y, r, S, t)=-\mathbb{E}_{t}^{\mathbb{Q}}\left[\int_{t}^{T} \mathrm{e}^{-\int_{t}^{u} r_{s} d s} \xi_{i}(u) d u\right] .
$$

As was proven in section 3.2, the indifference price increases in $\gamma$. Thus, $\widehat{H}^{(0)} \leq H$ is always a lower bound as stated in Corollary 6.1.

Next, we use the above asymptotic expansions to find approximate values for the optimal $\alpha^{\gamma}$ in (12) and $p^{\gamma}$ in (41) for small values of risk-aversion $\gamma$. To this end, we will explicitly indicate how the first two functions in the expansion depend on $\alpha$ :

$$
\widehat{H}^{(0)}=: \widehat{H}^{(0,0)}+\alpha \widehat{H}^{(0,1)}, \quad \widehat{H}^{(1)}=: \widehat{H}^{(1,0)}+\alpha \widehat{H}^{(1,1)}+\alpha^{2} \widehat{H}^{(1,2)},
$$

Plugging-in, we recover (38) and then in turn the approximation of (39) to $\alpha^{\gamma}$ for small values of $\gamma$.

Finally, for the relative indifference price $H^{\alpha A \mid \beta I}$ we obtain

$$
\begin{aligned}
H^{\alpha A \mid \beta I} & ={ }^{0} \widehat{H}^{\alpha A+\beta I}-{ }^{0} \widehat{H}^{\beta I}+\gamma\left({ }^{1} \widehat{H}^{\alpha A+\beta I}-{ }^{1} \widehat{H}^{\beta I}\right)+\mathcal{O}\left(\gamma^{2}\right) \\
& ={ }^{0} \widehat{H}^{\alpha A}+\gamma^{1} \widehat{H}^{\alpha A \mid \beta I}+\gamma^{2}\left({ }^{2} \widehat{H}^{\alpha A+\beta I}-{ }^{2} \widehat{H}^{\beta I}\right)+\mathcal{O}\left(\gamma^{3}\right)
\end{aligned}
$$


since the zero-order risk-neutral prices are once again additive with respect the contract payoff. The first term is ${ }^{0} \widehat{H}^{\alpha A}=\mathbb{E}_{t}^{\mathbb{Q}}\left[\int_{t}^{T} F(r, t ; u) \alpha A S_{u} d u\right]=\alpha \widehat{H}^{(0,1)}$ and the correction term solves

$$
{ }^{1} \widehat{H}_{t}^{\alpha A \mid \beta I}+\mathcal{L}_{H}{ }^{1} \widehat{H}^{\alpha A \mid \beta I}=-\frac{\sigma^{2}}{2 F\left(r_{t}, t ; T\right)}\left(\left({ }^{0} \widehat{H}_{y}^{\alpha A}\right)^{2}+2^{0} \widehat{H}_{y}^{\alpha A} \cdot{ }^{0} \widehat{H}_{y}^{\beta I}\right) .
$$

Comparing with (38) we find ${ }^{1} \widehat{H}^{\alpha A \mid \beta I}=\alpha \widehat{H}^{(1,1)}+\alpha^{2} \widehat{H}^{(1,2)}$.

\section{Proof of Theorem 7.1}

Proof: Substituting the $\delta$-expansion into (47) we get

$$
\begin{aligned}
D_{t}+\mathcal{L}_{r}^{\mathbb{Q}} D+\delta \widetilde{\mathcal{L}}_{Y} D+\delta \frac{\gamma}{F}\left(\frac{1}{2}\left(\sigma^{A}\right)^{2}\left(D_{y_{A}}\right)^{2}+\frac{1}{2}\left(\sigma^{I}\right)^{2}\left(D_{y_{I}}\right)^{2}+\rho \sigma^{A} \sigma^{I} \frac{\gamma}{F} D_{y_{A}} D_{y_{I}}\right) \\
+\widetilde{\mathcal{L}}_{S} D+f-r D=0 .
\end{aligned}
$$

Let us now consider an expansion in powers of $\delta: D=D^{(0)}+\delta D^{(1)}+\cdots$ Substituting this expansion into (A.88) and collecting terms yields that $D^{(i)}, i \in\{0,1\}$ solve

$$
u_{t}+\mathcal{L}_{r}^{\mathbb{Q}} u+\widetilde{\mathcal{L}}_{S} u-r u=-\xi_{i}, \quad u\left(y_{A}, y_{I}, r, S_{A}, S_{I}, T\right)=0
$$

where

$\xi_{0}(t)=f, \quad \xi_{1}(t)=\widetilde{\mathcal{L}}_{Y} D^{(0)}+\frac{\gamma}{F\left(r_{t}, t ; T\right)}\left(\frac{1}{2}\left\{\left(\sigma^{A}\right)^{2}\left(D_{y_{A}}^{(0)}\right)^{2}+\left(\sigma^{I}\right)^{2}\left(D_{y_{I}}^{(0)}\right)^{2}\right\}+\rho \sigma^{A} \sigma^{I} D_{y_{A}}^{(0)} D_{y_{I}}^{(0)}\right)$,

The solution of these pdes can be represented as

$$
\begin{aligned}
D^{(0)}= & \int_{t}^{T} F(r, t ; u) \cdot f\left(\Lambda^{A}\left(u, y_{A}\right), \Lambda^{I}\left(u, y_{I}\right), \mathrm{e}^{-\int_{t}^{u} \Lambda^{A}\left(s, y_{A}\right) d s} S^{A}, \mathrm{e}^{-\int_{t}^{u} \Lambda^{I}\left(s, y_{I}\right) d s} S^{I}\right) d u ; \quad \text { (A.90) } \\
D^{(1)}= & \mathbb{E}^{\mathbb{Q}}\left[\int_{t}^{T} \mathrm{e}^{-\int_{t}^{u} r_{s} d s}\left\{\widetilde{\mathcal{L}}_{Y} D^{(0)}+\frac{\gamma}{F\left(r_{u}, u ; T\right)}\left(\frac{\left(\sigma^{A}\right)^{2}}{2}\left(D_{y_{A}}^{(0)}\right)^{2}+\frac{\left(\sigma^{I}\right)^{2}}{2}\left(D_{y_{I}}^{(0)}\right)^{2}\right)\right\} d u \mid r_{t}=r\right] \\
& +\rho \mathbb{E}^{\mathbb{Q}}\left[\int_{t}^{T} \mathrm{e}^{-\int_{t}^{u} r_{s} d s} \frac{\gamma}{F\left(r_{u}, u ; T\right)} \sigma^{A} \sigma^{I} D_{y_{A}}^{(0)} D_{y_{I}}^{(0)} d u \mid r_{t}=r\right] .
\end{aligned}
$$

In the first expectation the argument is $D^{(0)} \equiv D^{(0)}\left(y_{0}^{A}, y_{0}^{I}, r, S^{A}, S^{I}, t\right)$, and it corresponds to deterministic mortality rates. In the second expectation the only random component is the interest rate $r_{u}$; the arguments of the functions inside the expectation are

$$
\left(y_{0}^{A}, y_{0}^{I}, r_{u}, \mathrm{e}^{-\int_{t}^{u} \Lambda^{A}\left(s, y_{0}^{A}\right) d s} S^{A}, \mathrm{e}^{-\int_{t}^{u} \Lambda^{I}\left(s, y_{0}^{I}\right) d s} S^{I}, u\right)
$$

whereas the arguments of $D^{(1)}$ itself are $\left(y_{0}^{A}, y_{0}^{I}, r, S^{A}, S^{I}, t\right)$.

We will approximate solutions to (12) and (41) when $\delta>0$ is small. In order to establish our result we will explicitly show how $D^{(0)}$ and $D^{(1)}$ depend on $\alpha$ :

$$
D^{(0)}=: D^{(0,0)}+\alpha D^{(0,1)}, \quad D^{(1)}=: D^{(1,0)}+\alpha D^{(1,1)}+\alpha^{2} D^{(1,2)},
$$


in which $D^{(0,0)}$ and $D^{(0,1)}$ are explicitly given by $(48)$, whereas $D^{(1,0)}, D^{(1,1)}$ and $D^{(1,2)}$ solve

$$
D_{t}^{i}+\mathcal{L}_{r}^{\mathbb{Q}} D^{i}+\widetilde{\mathcal{L}}_{S} D^{i}-r D^{i}=\left\{\begin{array}{r}
-\mu^{I} D_{y_{I}}^{(0,0)}-\frac{1}{2}\left(\sigma^{I}\right)^{2}\left(D_{y_{I} y_{I}}^{(0,0)}+\frac{\gamma}{F\left(r_{t}, t ; T\right)}\left(D_{y_{I}}^{(0,0)}\right)^{2}\right), \text { when } i=(1,0) \\
-\mu^{A} D_{y_{A}}^{(0,1)}-\frac{1}{2}\left(\sigma^{A}\right)^{2} D_{y_{A} y_{A}}^{(0,1)}-\frac{\gamma \rho \sigma^{A} \sigma^{I}}{F\left(r_{t}, t ; T\right)} D_{y_{I}}^{(0,0)} D_{y_{A}}^{(0,1)}, \text { when } i=(1,1) \\
-\frac{\gamma}{F\left(r_{t}, t ; T\right.} \frac{1}{2}\left(\sigma^{A}\right)^{2}\left(D_{y_{A}}^{(0,1)}\right)^{2}, \text { when } i=(1,2),
\end{array}\right.
$$

with zero terminal conditions, cf. (A.84). Applying Feynman-Kac yields (49) as required. Now, an approximate value for the optimal $\alpha^{\delta}$ in (12) can be obtained as using (37) when one replaces $\widetilde{H}$ by $D$. Similarly, an approximate optimizer for (41) is obtained by replacing $\widetilde{H}$ by $D$ in (43).

\section{References}

E. Bayraktar and V. R. Young. Hedging life insurance with pure endowments. Insurance Math. Econom., 40(3):435-444, 2007a.

E. Bayraktar and V. R. Young. Minimizing probability of ruin and a game of stopping and control. Technical report, University of Michigan, 2007b. Available at http://arxiv.org/abs/0704.2244.

E. Bayraktar and V. R. Young. Pricing options in incomplete equity markets via the instantaneous Sharpe ratio. Annals of Finance, 2008. In Press, available at http://arxiv.org/abs/math.OC/0701650.

D. Becherer. Rational hedging and valuation of integrated risks under constant absolute risk aversion. Insurance Math. Econom., 33(1):1-28, 2003.

T. Björk. Arbitrage Theory in Continuous Time. Oxford University Press, Oxford, UK, 2004.

T. Björk and I. Slinko. Towards a general theory of good deal bounds. Review of Finance, 10: 221-260, 2006.

N. Bowers, H. Gerber, J. Hickman, D. Jones, and C. Nesbitt. Actuarial Mathematics. Society of Actuaries, Schaumburg, IL, 1997.

A. J. G. Cairns, D. Blake, and K. Dowd. Pricing framework for securitization of mortality risk. Technical report, Heriot-Watt University, 2004.

S. Cox and Y. Lin. Natural hedging of life and annuity mortality risks. North American Actuarial Journal, 11(3):1-15, 2007.

M. Dahl. Stochastic mortality in life insurance: Market reserves and mortality-linked insurance contracts. Insurance Math. Econom., 35(1):113-136, 2004.

M. Dahl and T. Møller. Valuation and hedging of life insurance liabilities with systematic mortality risk. Insurance Math. Econom., 39(2):193-217, 2006. 
D. Duffie and T. Zariphopoulou. Optimal investment with undiversifiable income risk. Mathematical Finance, 3:135-148, 1993.

L. C. Evans. Partial Differential Equations, volume 19 of Graduate Studies in Mathematics. American Mathematical Society, Providence, Rhode Island, 1998.

W. H. Fleming and R. W. Rishel. Deterministic and stochastic optimal control. Springer-Verlag, Berlin, 1975. Applications of Mathematics, No. 1.

W. H. Fleming and H. M. Soner. Controlled Markov processes and viscosity solutions, volume 25 of Applications of Mathematics (New York). Springer-Verlag, New York, 1993.

H. Gerber. A probabilistic model for (life) contingencies and a delta-free approach to contingency reserves. Transactions of the Society of Actuaries, 28:127-148, 1976.

V. Henderson. Explicit solutions to an optimal portfolio choice problem with stochastic income. J. Econom. Dynam. Control, 29(7):1237-1266, 2005.

I. Karatzas and S. E. Shreve. Brownian motion and stochastic calculus, volume 113 of Graduate Texts in Mathematics. Springer-Verlag, New York, second edition, 1991.

N. V. Krylov. Controlled diffusion processes, volume 14 of Applications of Mathematics. SpringerVerlag, New York, 1980. Translated from the Russian by A. B. Aries.

R. Lee and L. Carter. Modeling and forecasting U.S. mortality. Journal of the American Statistical Association, 87:659-675, 1992.

M. Ludkovski and V. R. Young. Indifference pricing of pure endowments and life annuities under stochastic hazard and interest rates. Insurance Math. Econom., in Press, 2007.

M. A. Milevsky and S. D. Promislow. Mortality derivatives and the option to annuitize. Insurance Math. Econom., 29(3):299-318, 2001.

H. Pham. Smooth solutions to optimal investment models with stochastic volatilities and portfolio constraints. Appl. Math. Optim., 46(1):55-78, 2002.

M. H. Protter and H. F. Weinberger. Maximum principles in differential equations. Prentice-Hall Inc., Englewood Cliffs, N.J., 1967.

D. F. Schrager. Affine stochastic mortality. Insurance Math. Econom., 38(1):81-97, 2006.

Swiss Re. Annuities: a private solution to longevity risk. Technical report, Zurich, Switzerland, 2007. sigma Insurance Report 3-07.

V. R. Young. Equity-indexed life insurance: Pricing and reserving using the principle of equivalent utility. North American Actuarial Journal, 7(1):68-86, 2003.

V. R. Young. Pricing life insurance under stochastic mortality via the instantaneous Sharpe ratio. Insurance Math. Econom., 2008. In Press, available at http://arxiv.org/abs/0705.1297. 